\title{
A UNIFIED ANALYSIS OF TRANSMISSION CONDITIONS FOR THIN CONDUCTING SHEETS IN THE TIME-HARMONIC EDDY CURRENT MODEL
}

\author{
K. SCHMIDT* AND A. CHERNOV ${ }^{\dagger}$
}

\begin{abstract}
We introduce tools for a unified analysis and a comparison of impedance transmission conditions (ITCs) for thin conducting sheets within the time-harmonic eddy current model in two dimensions. The first criterion is the robustness with respect to the frequency or skin depth, that means if they give meaningful results for small and for large frequencies or conductivities. As a second tool we study the accuracy for a range of sheet thicknesses and frequencies for a relevant example, and analyse finally their asymptotic order in different asymptotic regimes. For the latter we write all the ITCs in a common form and show how they can be realised within the finite element method.

Two new conditions which we call ITC-2-0 and ITC-2-1 are introduced in this article which appear in a symmetric form. They are derived by asymptotic expansions in the asymptotic regime of constant ratio between skin depth and thickness like those in [34]. We analyse these ITCs in comparison with the often used perfect electric boundary condition, the conditions by Levi-Civita [26, 36], the shielding element by Nakata et.al. [29], the thin layer impedance boundary conditions by Mayergoyz et.al. $[42,27]$ and a family of ITCs derived by asymptotic expansions in the asymptotic regime of constant shielding by Schmidt and Tordeux [37].

Our analysis shows the superiority of the transmission conditions derived by asymptotic expansions where especially the worst-case error level of the ITC-2-1 is remarkably lower than for all the other conditions.
\end{abstract}

Key words. Impedance Transmission Conditions, Thin Conducting Sheets, Asymptotic Expansions, Eddycurrent model

AMS subject classifications. 65N30, 35C20, 35J25, 41A60, 35B40, 78M30, 78M35

Introduction. For simulations of the eddy current problem with thin sheets by standard methods, like the finite element method, the smallness of the thin sheet is first a challenge for automatic mesh generators, which nowadays hardly support anisotropic cells. If anyhow anisotropic simplicies (triangles or tetrahedra in 3D) in the sheet are used, the condition number of the (whole) system matrix [41] increase significantly. The thin sheet might be meshed by tensor-product cells (quadrilaterals or hexahedrons in 3D) with almost right angles where both by mesh refinement or increase of the order of polynomial basis functions the accuracy can be systematically reduced, however, for the price of a remarkable computational expense. This is especially the case for a pronounced boundary layer behaviour (or skin effect) for not that small frequencies and sheet thicknesses. This established method will be used to compute reference solutions to compare with the approximative impedance transmission conditions. Exponential convergence independent on the frequency is achieved if instead of polynomial basis functions inside the thin sheet a so called optimal basis which consist of frequency adapted hyperbolic functions multiplied with polynomials is taken [33]. Another approach with hyperbolic functions is proposed in [43].

The computational effort can be reduced by using transmission conditions which shall mimic the behaviour of the thin sheet. Those transmission conditions are applied at an interface to which the thin sheet is reduced. The application of transmission conditions is attractive, as the interface can easily (and usually automatic) be resolved by edges in the finite element mesh, and anisotropic cells with their drawbacks are omitted. These transmission conditions are also known as impedance boundary conditions [21] (the interface is considered as two-sided boundary), as shielding elements [29], as shell models [24], or as shell elements [17].

However, the notion impedance boundary conditions (IBCs) is traditionally used for approximative conditions on an artificial boundary which replaces a sub-domain of a solid conductor.

* DFG-research center MATHEON and Institut für Mathematik, Technische Universität Berlin, 10623 Berlin, Germany (kersten.schmidt@math.tu-berlin.de).

${ }^{\dagger}$ Hausdorff Center for Mathematics, University of Bonn, 53115 Bonn, Germany (chernov@hcm.uni-bonn.de). 
They have been first proposed by Shchukin [40] and Leontovich [25], and improved by several authors $[39,1,20,14]$. More similar to transmission conditions for thin sheets are generalised impedance boundary conditions for conducting bodies with dielectric coatings which are derived for high orders $[15,4,2,19,5]$ and for coatings with a kink [8]. The simplest boundary condition for solid conductors is, however, the perfect electric (PEC) boundary condition, which is even used for thin conducting sheets. It is common sense that the PEC should be used if the sheet thickness is small in comparison to the skin depth.

Impedance transmission conditions for thin conducting sheets have mainly be derived by using special functions in thickness direction which are multiplied by (macroscopic) functions along the sheet. Assuming no variation in thickness direction Levi-Civita [26],[3, Sec. 1.9],[38] has already postulated in 1902 that the tangential component of the electrical field shall be considered to be continuous over the sheet surface whereas the tangential component of magnetic field has a jump proportional to the tangential component of the electric field, as well as the conductivity and the thickness of the sheet. These very simple conditions have been used in boundary integral equations [23, 35], also derived from volume integral equations [28], in a network approach [9] or with the finite element method [31, 32, 6, 22]. Schmidt and Tordeux [36] showed that these conditions originate as limit when the sheet thickness tends to zero while the product of frequency and conductivity is scaled reciprocally to the sheet thickness. With the same assumption of no electric field variation in thickness direction, but with further tangential derivatives, the shielding element [29] has been introduced. Taking two hyperbolic functions in thickness direction which respects the boundary layer behaviour the so called thin layer impedance boundary conditions were derived by Mayergoyz et.al. [42, 27] and other authors [17, 21]. Similar expressions with the surface divergence are proposed by Krähenbühl and Muller [24]. Note, that adopting the ideas in [24] similar conditions for the time-dependant eddy current model has been derived e.g. in [7].

We will revisit the often used PEC boundary condition, the shielding element of [29] and the thin layer impedance boundary conditions by Mayergoyz et.al. [27], where the latter two turn out to be of order 0, i.e., the error (outside the sheet) decreases linearly with the sheet thickness.

The problems for thin conducting sheets differ from thin layers on the boundary by the fact that they are in the interior of the domain of interest and can not be approximated together with boundary conditions for solid bodies. As a consequence there are two interfaces of the thin sheet with the surrounding domain and setting a transmission condition derived for the two distinct interfaces on a single one, e. g., the mid-line, a modelling error proportional to the thickness [33] is achieved. Thus, those transmission conditions with even more than the two functions underlying the Krähenbühl-Muller conditions, e.g., in [18], are of order 0.

An asymptotic expansion of the solution for thin (and smooth) conducting sheets in an asymptotic framework in which the frequency is scaled reciprocally with the thickness is shown for any order by Schmidt and Tordeux [36]. This particular asymptotics was motivated by their non-trivial limit solution. Asymptotic expansion for thin isolating sheets with slowly or fast varying thickness of order 1 have been derived in $[30,11]$. In these asymptotic expansions the solution in the sheet is expanded in scaled coordinates, and the continuity conditions at the two interfaces of the sheet are expanded around its mid-line to obtain problems for the limit solution and corrector functions on the domain where the sheet is replaced by an interface. For the thin conducting sheets the asymptotic expansions of [36] have been used to to derive and verify transmission conditions up to order 3 in this framework [37]. With the same techniques but including the asymptotics of a frequency scaled reciprocally to the squared thickness transmission conditions up to order 1 have been derived in [34].

The transmission conditions derived by asymptotic expansions include in a systematic way the terms which are comparable larger than other terms which are excluded. So, by construction they are the optimal conditions in their own asymptotic regime, but it remains not fully clear how 
accurate are they for concrete sheet thicknesses and frequencies.

After introducing the time-harmonic eddy current model in two dimensions, the shielding efficiency and the skin depth in Section 1 eight impedance transmission conditions are introduced in Section 2, where two of them will be shown the first time in this article. In Section 3 the ITCs are analysed and compared with respect to their robustness in the frequency, their accuracy for a range of thicknesses and frequencies for a relevant example as well as their asymptotic order in different asymptotic regimes. The article is closed by a discussion in Section 4 .

1. Shielding by thin conducting sheets. We consider the time-harmonic eddy current model (with time convention $\mathrm{e}^{-\mathrm{i} \omega t}$ and the angular frequency $\omega>0$ ) in two dimensions

$$
\begin{aligned}
& \operatorname{curl}_{2 D} e(\mathbf{x})=\mathrm{i} \omega \mu_{0} \mathbf{h}(\mathbf{x}), \\
& \operatorname{curl}_{2 D} \mathbf{h}(\mathbf{x})=\sigma(\mathbf{x}) e(\mathbf{x})+j_{0}(\mathbf{x})
\end{aligned}
$$

where $e$ and $\mathbf{h}$ are the out-of-plane electric and in-plane magnetic fields, $\mathbf{x}=(x, y)^{\top}, \sigma$ is the conductivity and $j_{0}$ is the out-of-plane imposed current which is outside the conductor. We have used the $2 \mathrm{D}$ rotation operators $\operatorname{curl}_{2 D}=\left(\partial_{y},-\partial_{x}\right)^{\top}$ and $\operatorname{curl}_{2 D}=\left(-\partial_{y}, \partial_{x}\right)$. Usually the eddy current model (1.1) is considered only in a bounded domain $\Omega$ and appropriate boundary conditions are applied on $\partial \Omega$ to close the problem.

To obtain a variational formulation for the electric field only, we multiply (1.1b) with $\mathrm{i} \omega \mu_{0} e^{\prime}$ for some test function $e^{\prime}$ and use the integration by parts formula for $\operatorname{curl}_{2 D}$ and get with the tangential vector $\mathbf{n}^{\perp}$ on $\partial \Omega$

$$
\int_{\Omega} \mathrm{i} \omega \mu_{0} \sigma e e^{\prime}+\mathrm{i} \omega \mu_{0} j_{0} e^{\prime} \mathrm{d} \mathbf{x}=\mathrm{i} \omega \mu_{0} \int_{\Omega} \operatorname{curl}_{2 D} \mathbf{h} e^{\prime} \mathrm{d} \mathbf{x}=\int_{\Omega} \mathrm{i} \omega \mu_{0} \mathbf{h} \cdot \operatorname{curl}_{2 D} e^{\prime} \mathrm{d} \mathbf{x}-\mathrm{i} \omega \mu_{0} \int_{\partial \Omega} \mathbf{h} \cdot \mathbf{n}^{\perp} e^{\prime} \mathrm{d} \sigma(\mathbf{x}) .
$$

Now, inserting (1.1a) and assuming for simplicity a perfect electric conductor (PEC) boundary condition on the domain boundary $\partial \Omega$ we get

$$
\int_{\Omega} \operatorname{curl}_{2 D} e \cdot \operatorname{curl}_{2 D} e^{\prime}-\mathrm{i} \omega \mu_{0} \sigma e e^{\prime} \mathrm{d} \mathbf{x}=\mathrm{i} \omega \mu_{0} \int_{\Omega} j_{0} e^{\prime} \mathrm{d} \mathbf{x} .
$$

Note, that due to the identity $\operatorname{curl}_{2 D} e \cdot \operatorname{curl}_{2 D} e^{\prime}=\operatorname{grad} e \cdot \operatorname{grad} e^{\prime}$ we may always work with gradients instead.

We consider for simplicity of the presentation a single thin conducting $\Omega_{\text {int }}^{d}$, which is assumed to be smooth and its minimal radius of curvature is considerably larger than $d$. We denote $\Gamma$ its mid-line, $\mathbf{n}$ the normal unit vector on $\Gamma$ and the sheet surface $\partial \Omega_{\text {int }}^{d}$. The conductivity inside the thin sheet shall take a constant value $\sigma$. The exterior of $\Omega_{\text {int }}^{d}$ shall be $\Omega_{\text {ext }}^{d}$, and we assume that we have a non-conducting region on both sides of the sheet which is significantly thicker than $d$.

The induced currents $\sigma e$ in the thin sheets leads to diminished fields behind the thin conductor. This shielding effect may be measured on observation points $\mathbf{x}_{j}$ in $\Omega_{\text {ext }}^{d}$ by the shielding efficiency [10] (measured in decibels (dB))

$$
S E\left(\mathbf{x}_{j}\right)=20 \log _{10} \frac{\left|\mathbf{h}_{0}\left(\mathbf{x}_{j}\right)\right|}{\left|\mathbf{h}\left(\mathbf{x}_{j}\right)\right|},
$$

where $\mathbf{h}_{0}$ is the magnetic field in absence of a conducting sheet, i. e., $\sigma \equiv 0 \mathrm{in} \Omega_{\text {int }}^{d}$. If the frequency and the sheet thickness are not too small, then the induced currents flow mainly in a boundary layer of the sheet, i. e., on its skin. This effect is called skin effect where for thin sheets the relevant quantity is skin depth [13] of solid conductors

$$
d_{\text {skin }}:=\sqrt{\frac{2}{\omega \mu_{0} \sigma}}
$$

and its relation with the sheet thickness $\delta:=d_{\text {skin }} / d$. 
(a)

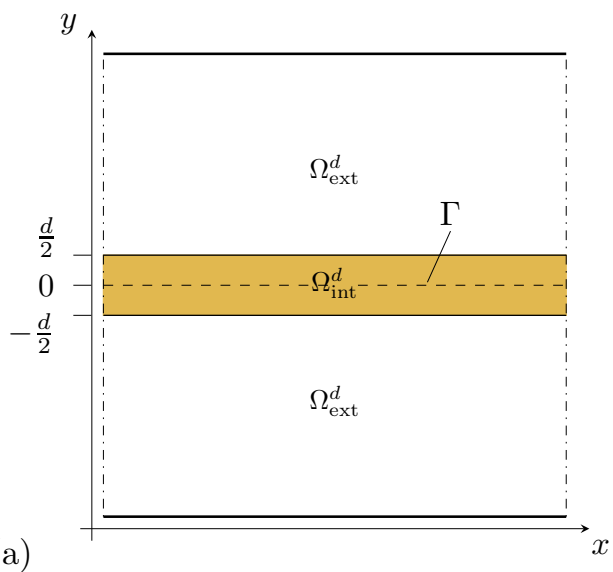

(b)

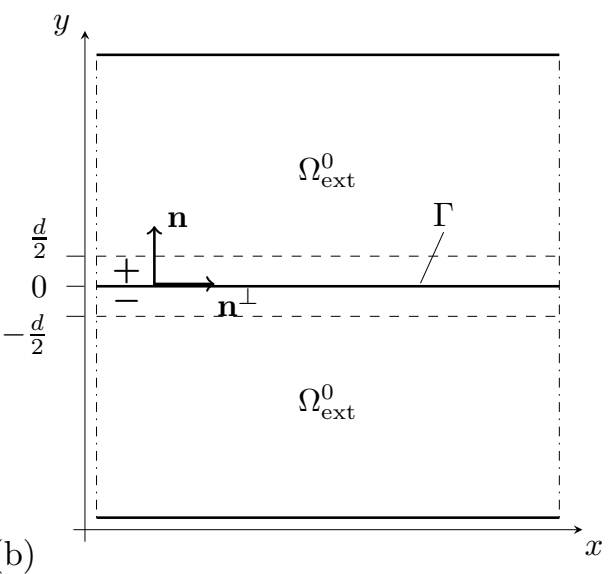

FIG. 1.1. (a) The geometrical setting of the original problem for the example of a straight thin sheet of thickness $d$. (b) The impedance transmission conditions are applied on the mid-line $\Gamma$ and we have a by non-conducting media extended "exterior domain" $\Omega_{\mathrm{ext}}^{0}$. The two sides for the definition of the jump and mean values of magnetic and electric field are indicated by "+" and "-".

\section{Overview of impedance transmission conditions for thin conducting sheets.}

2.1. The geometric setting of impedance transmission conditions in general. With the use of impedance transmission conditions the thin sub-domain $\Omega_{\text {int }}^{d}$ has not to be resolved by elements within a finite element discretisation or other numerical scheme. For this the exterior sub-domain is extended up to the mid-line $\Gamma$, see Fig. 1.1, where the conductivity close to $\Gamma$ is set to zero. The mid-line $\Gamma$ is now an interface for two sub-domains of the extented exterior domain $\Omega_{\text {ext }}^{0}$, over which the electric or magnetic field must not be continuous, i.e., we may have two distinct field values on both sides. The impedance transmission conditions are conditions between jumps and mean values of the tangential components of the electric and magnetic fields. If we signify the field values on the side the normal vector $\mathbf{n}$ points with a superscript "+" and and those on the other side with "-" (see Fig. 1.1(b)), and label with a subscript "ITC" the approximate solution for some impedance transmission conditions, then we denote the jumps by

$$
\left[e_{\mathrm{ITC}}\right]\left(\mathbf{x}_{\Gamma}\right)=e_{\mathrm{ITC}}^{+}\left(\mathbf{x}_{\Gamma}\right)-e_{\mathrm{ITC}}^{-}\left(\mathbf{x}_{\Gamma}\right), \quad\left[\mathbf{h}_{\mathrm{ITC}} \cdot \mathbf{n}^{\perp}\right]\left(\mathbf{x}_{\Gamma}\right)=\left(\mathbf{h}_{\mathrm{ITC}}^{+}\left(\mathbf{x}_{\Gamma}\right)-\mathbf{h}_{\mathrm{ITC}}^{-}\left(\mathbf{x}_{\Gamma}\right)\right) \cdot \mathbf{n}^{\perp}\left(\mathbf{x}_{\Gamma}\right),
$$

and the mean values by

$$
\left\{e_{\mathrm{ITC}}\right\}\left(\mathbf{x}_{\Gamma}\right)=\left(e_{\mathrm{ITC}}^{+}\left(\mathbf{x}_{\Gamma}\right)+e_{\mathrm{ITC}}^{-}\left(\mathbf{x}_{\Gamma}\right)\right) / 2, \quad\left\{\mathbf{h}_{\mathrm{ITC}} \cdot \mathbf{n}^{\perp}\right\}\left(\mathbf{x}_{\Gamma}\right)=\left(\mathbf{h}_{\mathrm{ITC}}^{+}\left(\mathbf{x}_{\Gamma}\right)+\mathbf{h}_{\mathrm{ITC}}^{-}\left(\mathbf{x}_{\Gamma}\right)\right) \cdot \mathbf{n}^{\perp}\left(\mathbf{x}_{\Gamma}\right) / 2,
$$

where $\mathbf{n}^{\perp}=\left(n_{2},-n_{1}\right)$ is the tangential unit vector on $\Gamma$. The line element for integration along $\Gamma$ is denoted by $\mathrm{dx}_{\Gamma}, \partial_{\Gamma}$ is the derivative in direction $\Gamma$ and $\kappa$ the signed curvature such that $\partial_{\Gamma} \mathbf{n}=\kappa \mathbf{n}^{\perp}$. With the interface parametrisation each point in the sheet can be written as $\mathbf{x}\left(s, \mathbf{x}_{\Gamma}\right)=\mathbf{x}_{\Gamma}+s \mathbf{n}\left(\mathbf{x}_{\Gamma}\right)$, $s \in\left[-\frac{d}{2}, \frac{d}{2}\right]$.

Let us enlist in the following some impedance transmission conditions we will study in this paper.

2.2. A list of impedance transmission conditions. In this section we are going to enlist several impedance transmission conditions, where we will use the compact notation for jumps and mean values except for some cases for a direct comparison with original articles.

The perfect electric conductor boundary condition is used, if strong shielding is assumed. The shielding element is thought to use for small thickness to skin depth ratios, where the conditions 
by Mayergoyz et.al., often called as thin sheet impedance boundary conditions, adapts to the skin depth and shall be applicable whatever is the thickness to skin depth ratio. The conditions by Levi-Civita [26, 3] correspond to the member ITC-1-0 in the family of transmission conditions ITC-1-N derived by asymptotic expansions. By asymptotic expansions two families of impedance transmission conditions are derived, whose members we denote by ITC-1-N or ITC-2-N, respectively. The first index corresponds to the scaling of $\omega \sigma$ with the varying thickness $\varepsilon$ inside the thin sheet and the second index $\mathrm{N}$ to the order starting with 0 . The conditions ITC-1-N are derived for the asymptotic regime $\omega \sigma \sim 1 / \varepsilon$ corresponding to asymptotically constant shielding and the conditions ITC-2-N for $\omega \sigma \sim 1 / \varepsilon^{2}$ corresponding to asymptotically constant relative skin depth. See Sec. 3.3 for more details on the asymptotic expansions.

2.2.1. Perfect electric conductor (PEC) boundary condition. The simplest conditions which are expected to give reasonable results for rather thick conductors of high conductivity or for high frequency are the PEC boundary condition, which sets the electric field on both sides to zero

$$
e_{\mathrm{PEC}}^{ \pm}\left(\mathbf{x}_{\Gamma}\right)=0
$$

In fact, it represents the asymptotic limit of vanishing thickness $d$ where at the same time $\sigma \omega / d \rightarrow \infty$.

2.2.2. Shielding element by Nakata et.al. [29]. The so called "shielding element" was introduced in [29], which denotes additional terms in a finite element formulation assuming a continuous electric field over $\Gamma$. To explain the shielding element we start with the variational formulation for the exact electric field $e$ which is

$$
\int_{\Omega_{\mathrm{ext}}^{d}} \operatorname{curl}_{2 D} e \cdot \operatorname{curl}_{2 D} e^{\prime} \mathrm{d} \mathbf{x}+\int_{\Omega_{\mathrm{int}}^{d}} \operatorname{curl}_{2 D} e \cdot \mathbf{c u r l}_{2 D} e^{\prime}-\mathrm{i} \omega \mu_{0} \sigma e e^{\prime} \mathrm{d} \mathbf{x}=\mathrm{i} \omega \mu_{0} \int_{\Omega_{\mathrm{ext}}^{d}} j_{0} e^{\prime} \mathrm{d} \mathbf{x},
$$

if we assume for simplicity PEC boundary conditions on $\partial \Omega$, no conductors outside the sheet and $e^{\prime}$ to be suitable test functions. As a first step of dimension reduction, with the extension of the exterior solution $e_{\text {NTFS }}$ up to the interface $\Gamma$ the first integral is extended to $\Omega_{\text {ext }}^{0}$ as well. Second, the field inside the sheet is assumed to be constant and can be represented by the function $e_{\text {NTFS }}$ on $\Gamma$, the derivatives in normal direction disappear and the integral in normal direction simplifies to a multiplication by $d$. Hence, the electric field for the "shielding element" is solution of

$$
\int_{\Omega_{\mathrm{ext}}^{0}} \operatorname{curl}_{2 D} e_{\mathrm{NTFS}} \cdot \mathbf{c u r l}_{2 D} e^{\prime} \mathrm{d} \mathbf{x}+d \int_{\Gamma} \partial_{\Gamma} e_{\mathrm{NTFS}} \partial_{\Gamma} e^{\prime}-\mathrm{i} \omega \mu_{0} \sigma e_{\mathrm{NTFS}} e^{\prime} \mathrm{d} \mathbf{x}_{\Gamma}=\mathrm{i} \omega \mu_{0} \int_{\Omega_{\mathrm{ext}}^{0}} j_{0} e^{\prime} \mathrm{d} \mathbf{x} .
$$

With the magnetic field $\mathbf{h}_{\text {NTFS }}$ which satisfies (1.1a) the electric field $e_{\text {NTFS }}$ fulfils (1.1b) (with $\sigma \equiv 0)$ as well. If we compare with the integration by parts formula

$$
\begin{aligned}
\mathrm{i} \omega \mu_{0} \int_{\Gamma}\left(\mathbf{h}_{\mathrm{NTFS}}^{-} \cdot \mathbf{n}^{\perp}-\mathbf{h}_{\mathrm{NTFS}}^{+} \cdot \mathbf{n}^{\perp}\right) e^{\prime} \mathrm{d} \mathbf{x}_{\Gamma} & =\mathrm{i} \omega \mu_{0} \int_{\Omega_{\text {ext }}^{0}} \mathbf{h}_{\mathrm{NTFS}} \cdot \operatorname{curl}_{2 D} e^{\prime}-\operatorname{curl}_{2 D} \mathbf{h}_{\mathrm{NTFS}} e^{\prime} \mathrm{d} \mathbf{x} \\
& =\int_{\Omega_{\mathrm{ext}}^{0}} \operatorname{curl}_{2 D} e_{\mathrm{NTFS}} \cdot \operatorname{curl}_{2 D} e^{\prime}-\mathrm{i} \omega \mu_{0} j_{0} e^{\prime} \mathrm{d} \mathbf{x},
\end{aligned}
$$

where we replaced $\mathbf{h}_{\mathrm{NTFS}}$ and $\operatorname{curl}_{2 D} \mathbf{h}_{\mathrm{NTFS}}$ using (1.1), to (2.2) and using the integrations by parts formula along the sheet mid-line $\Gamma$

$$
\int_{\Gamma} \partial_{\Gamma} e_{\mathrm{NTFS}} \partial_{\Gamma} e^{\prime} \mathrm{d} \mathbf{x}_{\Gamma}=-\int_{\Gamma} \partial_{\Gamma}^{2} e_{\mathrm{NTFS}} e^{\prime} \mathrm{d} \mathbf{x}_{\Gamma}
$$


we observe that the "shielding element" is equivalent to the impedance transmission conditions

$$
\begin{gathered}
e_{\mathrm{NTFS}}^{+}=e_{\mathrm{NTFS}}^{-}=: e_{\mathrm{NTFS}} \\
\mathbf{h}_{\mathrm{NTFS}}^{+} \cdot \mathbf{n}^{\perp}-\mathbf{h}_{\mathrm{NTFS}}^{-} \cdot \mathbf{n}^{\perp}=-d\left(\sigma+\frac{\partial_{\Gamma}^{2}}{\mathrm{i} \omega \mu_{0}}\right) e_{\mathrm{NTFS}}
\end{gathered}
$$

The approximative electric field inside the conducting sheet is for all $s \in\left[-\frac{d}{2}, \frac{d}{2}\right]$ the value on the interface

$$
e_{\mathrm{NTFS}, \text { int }}\left(\mathbf{x}_{\Gamma}+s \mathbf{n}\right)=e_{\mathrm{NTFS}}\left(\mathbf{x}_{\Gamma}\right) .
$$

2.2.3. Thin layer impedance boundary conditions by Mayergoyz-Bedrosian [27]. The thin layer impedance boundary conditions, sometimes called shell element, have been introduced for thin conducting sheets by Krähenbühl and Muller [24] where curvature effects are neglected. With an ansatz of two hyperbolic functions in thickness direction they relate the surface divergence of either the sum or the difference of the tangential component of the magnetic field to the difference or sum of its normal components. Using the same ansatz Mayergoyz and Bedrosian [27] derived relations between the jumps and mean values of electric and magnetic fields which are derived again in a nice way in [21]. In this section we are going to derive these condition step by step to observe the different sources of error. For this we assume a straight sheet as in Fig. 1.1. The transmission conditions are based on the assumption, that the field varies much less along the sheet than in its lateral direction. Consequently, the derivatives in longitudinal direction, which are here the $x$-derivatives, can be neglected and we get an ordinary differential equation (ODE) for the (approximative) electric field inside the sheet

$$
-\partial_{y}^{2} e_{\mathrm{MB}, \text { int }}(x, y)-\mathrm{i} \omega \mu_{0} \sigma e_{\mathrm{MB}, \text { int }}(x, y)=0 .
$$

To obtain transmission conditions on the common interface $\Gamma$ the simple condition

$$
e_{\mathrm{MB}}^{ \pm}(x, 0)=e_{\mathrm{MB}, \text { int }}\left(x, \pm \frac{d}{2}\right), \quad \quad \mathbf{h}_{\mathrm{MB}}^{ \pm}(x, 0) \cdot \mathbf{n}^{\perp}=\mathbf{h}_{\mathrm{MB}, \text { int }}\left(x, \pm \frac{d}{2}\right) \cdot \mathbf{n}^{\perp},
$$

where $e_{\mathrm{MB}}^{ \pm}$and $\mathbf{h}_{\mathrm{MB}}^{ \pm}$are the electric and magnetic fields on both sides of the interface. Taking the first condition serves as a boundary condition for (2.5) we can express the electric and magnetic field in the sheet by,

$$
\begin{aligned}
e_{\mathrm{MB}, \text { int }}(x, y) & =\left\{e_{\mathrm{MB}}\right\}(x) \frac{\cosh (\gamma y)}{\cosh \left(\gamma \frac{d}{2}\right)}+\left[e_{\mathrm{MB}}\right](x) \frac{\sinh (\gamma y)}{2 \sinh \left(\gamma \frac{d}{2}\right)} \\
\frac{1}{\gamma} \partial_{y} e_{\mathrm{MB}, \text { int }}(x, y)=\frac{\beta}{\gamma} \mathbf{h}_{\mathrm{MB}, \text { int }}(x, y) \cdot \mathbf{n}^{\perp} & =\left\{e_{\mathrm{MB}}\right\}(x) \frac{\sinh (\gamma y)}{\cosh \left(\gamma \frac{d}{2}\right)}+\left[e_{\mathrm{MB}}\right](x) \frac{\cosh (\gamma y)}{2 \sinh \left(\gamma \frac{d}{2}\right)}
\end{aligned}
$$

where $\gamma=\sqrt[+]{-\mathrm{i} \omega \mu_{0} \sigma}=(-1+\mathrm{i}) \sqrt{\omega \mu_{0} \sigma / 2}$ (note, that the sign of the square root is not important as $\gamma$ will be used only in even function) and $\beta=i \omega \mu_{0}$. Furthermore, note that the sign inside the square root of $\gamma$ is different to the definition in [21] as a different time convention is used.

Evaluating these formulas for $y= \pm \frac{d}{2}$ and using the approximative continuity conditions (2.6) we obtain the following relation between tangential components of electric and magnetic field on the two sheet surfaces

$$
\begin{aligned}
& e_{\mathrm{MB}}^{+}-e_{\mathrm{MB}}^{-}=\frac{\beta}{\gamma} \tanh \left(\gamma \frac{d}{2}\right)\left(\mathbf{h}_{\mathrm{MB}}^{+} \cdot \mathbf{n}^{\perp}+\mathbf{h}_{\mathrm{MB}}^{-} \cdot \mathbf{n}^{\perp}\right), \\
& \mathbf{h}_{\mathrm{MB}}^{+} \cdot \mathbf{n}^{\perp}-\mathbf{h}_{\mathrm{MB}}^{-} \cdot \mathbf{n}^{\perp}=\frac{\gamma}{\beta} \tanh \left(\gamma \frac{d}{2}\right)\left(e_{\mathrm{MB}}^{+} \quad+e_{\mathrm{MB}}^{-}\right),
\end{aligned}
$$

which are now meant in general coordinates and also for curved sheets. 
2.2.4. Thin layer impedance transmission conditions derived for asymptotically constant shielding. Thin layer impedance transmission conditions up to order 3 have been derived by Schmidt and Tordeux [37] by an asymptotic expansion of the fields in and outside the sheet (see [36]). Considering asymptotically small thicknesses $\varepsilon$ the asymptotic expansion techniques represents a systematic way to treat some terms as small compared to others. Writing the eddy current model for the ( $\varepsilon$-dependent) electric field $e_{\mathrm{int}}^{\varepsilon}$ inside the sheet $\Omega_{\mathrm{int}}^{\varepsilon}$

$$
\operatorname{curl}_{2 D} \operatorname{curl}_{2 D} e_{\text {int }}^{\varepsilon}(\mathbf{x})+\gamma_{1}^{2}(\varepsilon) e_{\text {int }}^{\varepsilon}(\mathbf{x})=0
$$

where the quantity $\gamma_{1}(\varepsilon)=\gamma \sqrt{d / \varepsilon}\left(\gamma=\sqrt{-\mathrm{i} \omega \mu_{0} \sigma}\right.$ was defined in Sect. 2.2.3) is scaled with the varying sheet thickness $\varepsilon$ such that the shielding behaviour does not essentially change with $\varepsilon$. This asymptotic behaviour is illustrated in Fig. 3.1 (right). The dash-dotted line corresponds to sheets of varying thickness $\varepsilon$ for which the skin depth $d_{\text {skin }}=\sqrt{\varepsilon \cdot 1.56 \mathrm{~mm}}$ where the shielding efficiency is close to $20.2 \mathrm{~dB}$. Note, that for $\varepsilon=d$ the model coincides with the original one. The asymptotic expansion inside the sheet is in normalised coordinates $S=\frac{s}{\varepsilon}$ motivated by the idea of self-similar solutions. Hence, the derivatives in normal direction scale like $\varepsilon^{-1}$ while those in tangential direction are unchanged. Sorting for same orders in $\varepsilon$ an ordinary differential equations in thickness direction is obtained, which is solved in general by a polynomial and for the lowest order conditions, like for the shielding element, it leads to a constant behaviour. The expansion of the electric field in the sheet $e_{\mathrm{int}}^{\varepsilon}$ is matched at the two sheet surfaces to the expansion of the exterior electric field $e_{\mathrm{ext}}^{\varepsilon}$

$$
e_{\text {ext }}^{\varepsilon}(\mathbf{x})=e_{\text {ext }}^{0}(\mathbf{x})+\varepsilon e_{\text {ext }}^{1}(\mathbf{x})+\varepsilon^{2} e_{\text {ext }}^{2}(\mathbf{x})+\ldots
$$

The expansion is constructed to obtain accurate approximations for any $\varepsilon \leq d$, which means especially that the terms of the expansion $e_{\text {ext }}^{j}$ have to be defined for any small $\varepsilon>0$ and so up to the interface $\Gamma$. Thus, the asymptotic expansion provides a natural extension of the exact solution up to the interface $\Gamma$, however, only approximately to some order in $\varepsilon$. For smooth sheets the terms $e_{\text {ext }}^{j}$ have enough regularity such that their values at the two sheets surfaces (at $s= \pm \frac{\varepsilon}{2}$ ) can be expanded using the Taylor expansion around the interface $\Gamma$ (at $s= \pm 0$ ). With this approach all contributions to the error can be reduced, at least for $\varepsilon$ small enough, by using a higher order model in $\varepsilon$.

Writing the derived transmission conditions in terms of the electric and magnetic field we have the impedance transmission conditions of order 0 (ITC-1-0) and the approximative electric field inside the conducting sheet

$$
\begin{aligned}
e_{\mathrm{ITC}, 1,0}^{+} & =e_{\mathrm{ITC}, 1,0}^{-}=: e_{\mathrm{ITC}, 1,0}, \\
\mathbf{h}_{\mathrm{ITC}, 1,0}^{+} \cdot \mathbf{n}^{\perp}-\mathbf{h}_{\mathrm{ITC}, 1,0}^{-} \cdot \mathbf{n}^{\perp} & =-d \sigma e_{\mathrm{ITC}, 1,0}, \\
e_{\mathrm{ITC}, 1,0, \text { int }}\left(\mathbf{x}_{\Gamma}+s \mathbf{n}\right) & =e_{\mathrm{ITC}, 1,0} .
\end{aligned}
$$

The transmission conditions and the internal electric field of order 1 (ITC-1-1) are given by

$$
\begin{aligned}
e_{\mathrm{ITC}, 1,1}^{+} & =e_{\mathrm{ITC}, 1,1}^{-}=: e_{\mathrm{ITC}, 1,1}, \\
\mathbf{h}_{\mathrm{ITC}, 1,1}^{+} \cdot \mathbf{n}^{\perp}-\mathbf{h}_{\mathrm{ITC}, 1,1}^{-} \cdot \mathbf{n}^{\perp} & =-d \sigma\left(1-\frac{\mathrm{i} \omega \mu_{0} \sigma d^{2}}{6}\right) e_{\mathrm{ITC}, 1,1}, \\
e_{\mathrm{ITC}, 1,1, \operatorname{int}}\left(\mathbf{x}_{\Gamma}+s \mathbf{n}\right) & =\left(1-\mathrm{i} \omega \mu_{0} \sigma\left(\frac{s^{2}}{2}+\frac{d^{2}}{8}\right)\right) e_{\mathrm{ITC}, 1,1}\left(\mathbf{x}_{\Gamma}\right)+\mathrm{i} \omega \mu_{0} s\left\{\mathbf{h}_{\mathrm{ITC}, 1,1} \cdot \mathbf{n}^{\perp}\right\}\left(\mathbf{x}_{\Gamma}\right) .
\end{aligned}
$$


As the transmission conditions of order 2 (ITC-1-2) are more complex we use for their definition the compact notation of jumps and mean values:

$$
\begin{aligned}
& {\left[e_{\mathrm{ITC}, 1,2}\right]=\quad d^{3} \frac{\mathrm{i} \omega \mu_{0} \sigma \kappa}{24} \quad\left\{e_{\mathrm{ITC}, 1,2}\right\}-d^{3} \frac{\omega^{2} \mu_{0}^{2} \sigma}{12}\left\{\mathbf{h}_{\mathrm{ITC}, 1,2} \cdot \mathbf{n}^{\perp}\right\},} \\
& {\left[\mathbf{h}_{\mathrm{ITC}, 1,2} \cdot \mathbf{n}^{\perp}\right]=-d \sigma\left(1-\frac{\mathrm{i} \omega \mu_{0} d^{2}}{6}+\frac{d^{2}}{12}\left(d^{2} \frac{7 \omega^{2} \mu_{0}^{2} \sigma^{2}}{20}+\partial_{\Gamma}^{2}\right)\right)\left\{e_{\mathrm{ITC}, 1,2}\right\}-d^{3} \frac{\mathrm{i} \omega \mu_{0} \sigma \kappa}{24}\left\{\mathbf{h}_{\mathrm{ITC}, 1,2} \cdot \mathbf{n}^{\perp}\right\} .}
\end{aligned}
$$

The respective internal electric field can be computed a-posteriori by

$$
\begin{aligned}
& e_{\mathrm{ITC}, 1,2, \operatorname{int}}\left(\mathbf{x}_{\Gamma}+s \mathbf{n}\right)= \\
& \left(1-\frac{1}{2} \mathrm{i} \omega \mu_{0} \sigma\left(s^{2}+\frac{d^{2}}{4}\right)+\frac{1}{6} \mathrm{i} \omega \mu_{0} \sigma \kappa\left(\mathbf{x}_{\Gamma}\right) s\left(s^{2}+\frac{3}{4} d^{2}\right)-\frac{1}{24} \omega^{2} \mu_{0}^{2} \sigma^{2}\left(s^{2}+\frac{3}{4} d^{2}\right)^{2}\right)\left\{e_{\mathrm{ITC}, 1,2}\right\}\left(\mathbf{x}_{\Gamma}\right) \\
& \quad+\mathrm{i} \omega \mu_{0} s\left(1-\frac{1}{2} \kappa\left(\mathbf{x}_{\Gamma}\right) s-\frac{1}{6} \mathrm{i} \omega \mu_{0} \sigma\left(s^{2}-\frac{3}{4} d^{2}\right)\right)\left\{\mathbf{h}_{\mathrm{ITC}, 1,2} \cdot \mathbf{n}^{\perp}\right\}\left(\mathbf{x}_{\Gamma}\right)-\frac{1}{2} s^{2}\left(\partial_{\Gamma}^{2}\left\{e_{\mathrm{ITC}, 1,2}\right\}\right)\left(\mathbf{x}_{\Gamma}\right) .
\end{aligned}
$$

The curvature of the thin sheet as well as the tangential derivatives are considered, they appear due to their asymptotic smallness, however, only from the transmission conditions from order 2 on.

2.2.5. Thin layer impedance transmission conditions derived for asymptotically constant relative skin depth. Similarly to the previous impedance transmission conditions, Schmidt and Chernov [34] have derived those in the asymptotics of constant relative skin-depth. This asymptotic framework corresponds to the eddy current model for the ( $\varepsilon$-dependent) electric field $e_{\mathrm{int}}^{\varepsilon}$ inside the sheet $\Omega_{\mathrm{int}}^{\varepsilon}$

$$
\operatorname{curl}_{2 D} \operatorname{curl}_{2 D} e_{\mathrm{int}}^{\varepsilon}(\mathbf{x})+\gamma_{2}^{2}(\varepsilon) e_{\mathrm{int}}^{\varepsilon}(\mathbf{x})=0
$$

where $\gamma_{2}(\varepsilon)=\gamma d / \varepsilon$. The limit for vanishing sheet thickness $d \rightarrow 0$ leads in this so called $\alpha=2$ asymptotic to the PEC boundary conditions already introduced in Sect. 2.2.1. The conditions of order 1 in the $\alpha=2$-asymptotics introduce both jumps of the electric and magnetic field, but for curved sheets in a non-symmetric form. Here, we propose two new impedance transmission conditions which differ each by one term from the order 1 conditions in [34], such that their variational formulations take both a symmetric form (see Sec. 2.3), respectively. We call ITC-2-0 the impedance transmission condition which has the same jump of the magnetic field as the above mentioned $\alpha=2$ conditions of order 1 of [34], but no jump in the electric field,

$$
\begin{aligned}
e_{\mathrm{ITC}, 2,0}^{+} & =e_{\mathrm{ITC}, 2,0}^{-}=: e_{\mathrm{ITC}, 2,0}, \\
\mathbf{h}_{\mathrm{ITC}, 2,0}^{+} \cdot \mathbf{n}^{\perp}-\mathbf{h}_{\mathrm{ITC}, 2,0}^{-} \cdot \mathbf{n}^{\perp} & =\frac{\gamma}{\beta} \frac{2 \sinh \left(\gamma \frac{d}{2}\right)}{\cosh \left(\gamma \frac{d}{2}\right)-\gamma \frac{d}{2} \sinh \left(\gamma \frac{d}{2}\right)} e_{\mathrm{ITC}, 2,0} .
\end{aligned}
$$

We remark that the transmission conditions have the same form as those in (2.12) and (2.13) whereas the constant consists of $\sinh \left(\gamma \frac{d}{2}\right)$ and $\cosh \left(\gamma \frac{d}{2}\right)$ like in $(2.9 \mathrm{~b})$. The respective internal electric field can be computed a-posteriori by

$$
e_{\mathrm{ITC}, 2,0, \text { int }}\left(\mathbf{x}_{\Gamma}+s \mathbf{n}\right)=\frac{\cosh (\gamma s)}{\cosh \left(\gamma \frac{d}{2}\right)-\gamma \frac{d}{2} \sinh \left(\gamma \frac{d}{2}\right)} e_{\mathrm{ITC}, 2,0}\left(\mathbf{x}_{\Gamma}\right) .
$$


To obtain order 1 transmission conditions we start from those for $\alpha=2$ in [34], but add a term in the jump condition for the magnetic field arising already from the conditions for $\alpha=2$ of order 2 which we derived in the same way as in [34]. We call these conditions ITC-2-1 which are given by

$$
\begin{aligned}
{\left[e_{\mathrm{ITC}, 2,1}\right] } & =d\left(1-\left(\gamma \frac{d}{2}\right)^{-1} \tanh \left(\gamma \frac{d}{2}\right)\right)\left(\beta\left\{\mathbf{h}_{\mathrm{ITC}, 2,1} \cdot \mathbf{n}^{\perp}\right\}+\frac{1}{2} \kappa\left\{e_{\mathrm{ITC}, 2,1}\right\}\right), \\
{\left[\mathbf{h}_{\mathrm{ITC}, 2,1} \cdot \mathbf{n}^{\perp}\right] } & =\frac{\gamma}{\beta} \frac{2 \sinh \left(\gamma \frac{d}{2}\right)}{\cosh \left(\gamma \frac{d}{2}\right)-\gamma \frac{d}{2} \sinh \left(\gamma \frac{d}{2}\right)}\left\{e_{\mathrm{ITC}, 2,1}\right\}-\frac{1}{2} d \kappa\left(1-\left(\gamma \frac{d}{2}\right)^{-1} \tanh \left(\gamma \frac{d}{2}\right)\right)\left\{\mathbf{h}_{\mathrm{ITC}, 2,1} \cdot \mathbf{n}^{\perp}\right\} .
\end{aligned}
$$

The respective internal electric field can be computed a-posteriori by

$$
\begin{aligned}
e_{\mathrm{ITC}, 2,1, \text { int }}\left(\mathbf{x}_{\Gamma}+s \mathbf{n}\right)= & \frac{\cosh (\gamma s)}{\cosh \left(\gamma \frac{d}{2}\right)-\gamma \frac{d}{2} \sinh \left(\gamma \frac{d}{2}\right)}\left(1-\frac{1}{2} s \kappa\left(\mathbf{x}_{\Gamma}\right)\right)\left\{e_{\mathrm{ITC}, 2,1}\right\}\left(\mathbf{x}_{\Gamma}\right) \\
& +\frac{\sinh (\gamma s)}{\gamma \cosh \left(\gamma \frac{d}{2}\right)}\left(\beta\left\{\mathbf{h}_{\mathrm{ITC}, 2,1} \cdot \mathbf{n}^{\perp}\right\}\left(\mathbf{x}_{\Gamma}\right)+\frac{1}{2} \kappa\left(\mathbf{x}_{\Gamma}\right)\left\{e_{\mathrm{ITC}, 2,1}\right\}\left(\mathbf{x}_{\Gamma}\right)\right) .
\end{aligned}
$$

2.3. Writing the ITCs in a general form for the unified analysis. For their unified analysis and the definition of their variational formulations we prefer to write the introduced impedance transmission conditions in the general form

$$
\begin{aligned}
& 0=T_{11}\left[e_{\mathrm{ITC}}\right]+T_{12}\left\{e_{\mathrm{ITC}}\right\}+T_{13}\left\{\operatorname{curl}_{2 D} e_{\mathrm{ITC}} \cdot \mathbf{n}^{\perp}\right\}, \\
& 0=T_{21}\left[\operatorname{curl}_{2 D} e_{\mathrm{ITC}} \cdot \mathbf{n}^{\perp}\right]+T_{22}\left\{e_{\mathrm{ITC}}\right\}+T_{23}\left\{\operatorname{curl}_{2 D} e_{\mathrm{ITC}} \cdot \mathbf{n}^{\perp}\right\},
\end{aligned}
$$

where we use the electric field only and where $T_{i j}=T_{i j}\left(d,-\mathrm{i} \omega \mu_{0} \sigma, \kappa ; \mathbf{x}_{\Gamma}\right)$ depend on $d, \mathrm{i} \omega \mu_{0} \sigma$ and the curvature $\kappa\left(\mathbf{x}_{\Gamma}\right)$. For example for the very simple PEC boundary conditions we have $T_{11}=T_{22}=1$ and $T_{i j}=0$ otherwise. The coefficients $T_{i j}$ for the introduced ITCs as well as for an absent sheet ("no sheet") are summarised in Tab. 2.1.

2.3.1. Variational formulation for continuous electric fields. For four of the introduced transmission conditions the (approximate) electric field is continuous over the interface $\left(T_{12}=\right.$ $T_{13}=0$ ), these are the shielding element, ITC-1-0, ITC-1-1 and ITC-2-0. We exclude the PEC in this section as the electric field is not only continuous but zero on $\Gamma$. For these ITCs the jump of the magnetic field is only dependent on the mean value of the electric field $\left(T_{23}=0\right)$ and $T_{21}=1$ which we assume in the following as well.

Assuming no conductivities except in the thin sheet the term with $\sigma$ in (1.2) vanishes, but integration by parts in the sub-domains of $\Omega$ separated by $\Gamma$ and inserting the transmission conditions leads to an integral term over $\Gamma$ instead.

As the magnetic field and so $\operatorname{curl}_{2 D} e_{\mathrm{ITC}} \cdot \mathbf{n}^{\perp}$ are not continuous over $\Gamma$ we get

$$
\int_{\Omega} \operatorname{curl}_{2 D} e_{\mathrm{ITC}} \cdot \operatorname{curl}_{2 D} e^{\prime} \mathrm{d} \mathbf{x}-\int_{\Gamma}\left[\operatorname{curl}_{2 D} e_{\mathrm{ITC}} \cdot \mathbf{n}^{\perp}\right]\left\{e^{\prime}\right\} \mathrm{d} \mathbf{x}_{\Gamma}=\mathrm{i} \omega \mu_{0} \int_{\Omega} j_{0} e^{\prime} \mathrm{d} \mathbf{x} .
$$

Inserting the transmission condition (2.18b) and with the identity $\operatorname{curl}_{2 D} e_{\mathrm{ITC}} \cdot \operatorname{curl}_{2 D} e^{\prime}=\operatorname{grad} e_{\mathrm{ITC}}$. $\operatorname{grad} e^{\prime}$ the variational formulation reads

$$
\int_{\Omega} \operatorname{grad} e_{\mathrm{ITC}} \cdot \operatorname{grad} e^{\prime} \mathrm{d} \mathbf{x}+\int_{\Gamma} T_{22}\left\{e_{\mathrm{ITC}}\right\}\left\{e^{\prime}\right\} \mathrm{d} \mathbf{x}_{\Gamma}=\mathrm{i} \omega \mu_{0} \int_{\Omega} j_{0} e^{\prime} \mathrm{d} \mathbf{x} .
$$

Note, that the bilinear form is symmetric. For the shielding element the second tangential derivative in $T_{22}$ is reduced by one in the variational formulation by using integration by parts along $\Gamma$. The formulation is well-posed for the shielding element, ITC-1-0 and ITC-1-1 as $\operatorname{Im} T_{22}=\omega \mu_{0} \sigma d>0$ and for ITC-2-0 one easily verifies that $\operatorname{Im} T_{22}>0$ for any $\sigma \omega>0$. 


\begin{tabular}{|c|c|c|c|}
\hline ITC & $T_{11}$ & $T_{12}$ & $T_{13}$ \\
\hline $\begin{array}{l}\text { PEC b.c. } \\
\text { No sheet }\end{array}$ & $\begin{array}{l}1 \\
1\end{array}$ & & \\
\hline Shielding element (NTFS) & 1 & & \\
\hline Thin layer IBC (MB) & 1 & & $-\frac{2}{\gamma} \tanh \left(\gamma \frac{d}{2}\right)$ \\
\hline ITC-1-0 & 1 & & \\
\hline ITC-1-1 & 1 & & \\
\hline ITC-1-2 & 1 & $-\kappa \frac{\gamma^{2} d^{3}}{24}$ & $\frac{\gamma^{2} d^{3}}{12}$ \\
\hline ITC-2-0 & 1 & & \\
\hline ITC-2-1 & 1 & $-\kappa \frac{d}{2}\left(1-\frac{2}{\gamma d} \tanh \left(\gamma \frac{d}{2}\right)\right)$ & $d\left(1-\frac{2}{\gamma d} \tanh \left(\gamma \frac{d}{2}\right)\right)$ \\
\hline ITC & $T_{21}$ & $T_{22}$ & $T_{23}$ \\
\hline $\begin{array}{l}\text { PEC b.c. } \\
\text { No sheet }\end{array}$ & 1 & 1 & \\
\hline Shielding element (NTFS) & 1 & $-\gamma^{2} d+d \partial_{\Gamma}^{2}$ & \\
\hline Thin layer IBC (MB) & 1 & $-2 \gamma \tanh \left(\gamma \frac{d}{2}\right)$ & \\
\hline ITC- $1-0$ & 1 & $-\gamma^{2} d$ & \\
\hline ITC-1-1 & 1 & $-\gamma^{2} d\left(1+\frac{\gamma^{2} d^{2}}{6}\right)$ & \\
\hline ITC-1-2 & 1 & $-\gamma^{2} d\left(1+\frac{\gamma^{2} d^{2}}{6}+\frac{d^{2}}{12}\left(\frac{-7 \gamma^{4} d^{2}}{20}+\partial_{\Gamma}^{2}\right)\right)$ & $\kappa \frac{\gamma^{2} d^{3}}{24}$ \\
\hline ITC-2-0 & 1 & $-\frac{2 \gamma \sinh \left(\gamma \frac{d}{2}\right)}{\cosh \left(\gamma \frac{d}{2}\right)-\gamma \frac{d}{2} \sinh \left(\gamma \frac{d}{2}\right)}$ & \\
\hline ITC-2-1 & 1 & $-\frac{2 \gamma \sinh \left(\gamma \frac{d}{2}\right)}{\cosh \left(\gamma \frac{d}{2}\right)-\gamma \frac{d}{2} \sinh \left(\gamma \frac{d}{2}\right)}$ & $\kappa \frac{d}{2}\left(1-\frac{2}{\gamma d} \tanh \left(\gamma \frac{d}{2}\right)\right)$ \\
\hline
\end{tabular}

TABLE 2.1

The coefficients $T_{i j}$ in the general form (2.18) for the introduced impedance boundary conditions, where $\gamma=$ $\sqrt{-\mathrm{i} \omega \mu_{0} \sigma}$. The zero coefficients are omitted for clarity. If there would be "no sheet" (the area is non-conducting) the exact conditions are specified.

\subsubsection{Primal variational formulation for thin layer impedance boundary condi-} tions, ITC-1-2 and ITC-2-1. If the electric field is not necessarily continuous over the interface $\Gamma$ we have instead of (2.19) the equality

$\int_{\Omega} \operatorname{curl}_{2 D} e_{\mathrm{ITC}} \cdot \operatorname{curl}_{2 D} e^{\prime} \mathrm{d} \mathbf{x}-\int_{\Gamma}\left[\operatorname{curl}_{2 D} e_{\mathrm{ITC}} \cdot \mathbf{n}^{\perp}\right]\left\{e^{\prime}\right\}+\left\{\operatorname{curl}_{2 D} e_{\mathrm{ITC}} \cdot \mathbf{n}^{\perp}\right\}\left[e^{\prime}\right] \mathrm{d} \mathbf{x}_{\Gamma}=\mathrm{i} \omega \mu_{0} \int_{\Omega} j_{0} e^{\prime} \mathrm{d} \mathbf{x}$.

For the thin layer impedance boundary condition, the ITC-1-2 and the ITC-2-1 the jump of the electric field depends on the mean value of its $\operatorname{curl}_{2 D} e_{\mathrm{ITC}} \cdot \mathbf{n}^{\perp}$, i.e., $T_{13} \neq 0$, and we can replace the latter using (2.18a) and $T_{11}=1$

$$
\left\{\operatorname{curl}_{2 D} e_{\mathrm{ITC}} \cdot \mathbf{n}^{\perp}\right\}=-T_{13}^{-1}\left[e_{\mathrm{ITC}}\right]-T_{12} T_{13}^{-1}\left\{e_{\mathrm{ITC}}\right\} .
$$


Inserting this equality in $(2.18 \mathrm{~b})$ we have

$$
\left[\operatorname{curl}_{2 D} e_{\mathrm{ITC}} \cdot \mathbf{n}^{\perp}\right]=T_{23} T_{13}^{-1}\left[e_{\mathrm{ITC}}\right]-\left(T_{22}-T_{23} T_{12} T_{13}^{-1}\right)\left\{e_{\mathrm{ITC}}\right\} .
$$

Hence, we get the variational formulation

$$
\begin{aligned}
\int_{\Omega} \operatorname{grad} e_{\mathrm{ITC}} \cdot \operatorname{grad} e^{\prime} \mathrm{d} \mathbf{x} & +\int_{\Gamma}\left(T_{22}-T_{23} T_{12} T_{13}^{-1}\right)\left\{e_{\mathrm{ITC}}\right\}\left\{e^{\prime}\right\}+T_{13}^{-1}\left[e_{\mathrm{ITC}}\right]\left[e^{\prime}\right] \\
& +T_{12} T_{13}^{-1}\left\{e_{\mathrm{ITC}}\right\}\left[e^{\prime}\right]-T_{23} T_{13}^{-1}\left[e_{\mathrm{ITC}}\right]\left\{e^{\prime}\right\} \mathrm{d} \mathbf{x}_{\Gamma}=\mathrm{i} \omega \mu_{0} \int_{\Omega} j_{0} e^{\prime} \mathrm{d} \mathbf{x}
\end{aligned}
$$

whose bilinear form is symmetric if $T_{12}=-T_{23}$. This is the case for all the three above mentioned ITCs. As above for the shielding element for ITC-1-2 the second tangential derivative in $T_{22}$ is reduced by one in the variational formulation by using the integration by parts formula (2.3) along $\Gamma$.

For the thin layer impedance boundary conditions the variational formulation is well-posed as $\operatorname{Im} T_{22}>0, \operatorname{Im} T_{13}^{-1}>0$ (for $\left.\omega \sigma>0\right)$ and $T_{12}=T_{23}=0$. One verifies as well for the thin layer impedance boundary conditions and for ITC-2-1 that $\operatorname{Im}\left(T_{22}-T_{12} T_{23} T_{13}^{-1}\right)>0$ and $\operatorname{Im} T_{13}^{-1}>0$ without any condition on the curvature or thickness.

2.3.3. Mixed variational formulation for any of the ITCs. Independently on the ITCs (except for PEC) we may replace $\left\{\operatorname{curl}_{2 D} e_{\mathrm{ITC}} \cdot \mathbf{n}^{\perp}\right\}$ by $-\lambda_{\text {ITC }}$ where $\lambda_{\text {ITC }}$ is a new variable. Inserting (2.18b) into (2.21), but adding (2.18a) as a separated equation we get the mixed variational formulation

$$
\begin{gathered}
\int_{\Omega} \operatorname{grad} e_{\mathrm{ITC}} \cdot \operatorname{grad} e^{\prime} \mathrm{d} \mathbf{x}+\int_{\Gamma} T_{22}\left\{e_{\mathrm{ITC}}\right\}\left\{e^{\prime}\right\}-T_{23} \lambda_{\mathrm{ITC}}\left\{e^{\prime}\right\}+\lambda_{\mathrm{ITC}}\left[e^{\prime}\right] \mathrm{d} \mathbf{x}_{\Gamma}=\mathrm{i} \omega \mu_{0} \int_{\Omega} j_{0} e^{\prime} \mathrm{d} \mathbf{x}, \\
\int_{\Gamma}\left[e_{\mathrm{ITC}}\right] \lambda^{\prime}+T_{12}\left\{e_{\mathrm{ITC}}\right\} \lambda^{\prime}-T_{13} \lambda_{\mathrm{ITC}} \lambda^{\prime} \mathrm{d} \mathbf{x}_{\Gamma}=0 .
\end{gathered}
$$

Here we used $T_{11}=T_{21}=1$ and $\lambda^{\prime}$ is a test function related to $\lambda$. The bilinear form of the mixed system is symmetric if $T_{12}=-T_{23}$ which is the case for all introduced impedance transmission conditions. Once again we use integration by parts on $\Gamma$ for any tangential second derivative in $T_{22}$.

3. Analysis of the impedance transmission conditions. In this section we are going to study the properties of the eight impedance transmission conditions introduced in the previous section. First, we analyse their robustness with respect to the frequency or skin depth, that means if they give meaningful results for small and for large conductivities or frequencies. Second, we study the dependence of the accuracy with respect to the thickness and the conductivity for straight sheet where we use the $h p$-finite element method to highly resolve the fields. Lastly, we study the transmissions conditions asymptotically for small thicknesses which we will compare with the asymptotic expansion of the exact fields.

3.1. Robustness of the impedance transmission conditions. The proposed impedance transmission conditions depend possibly on the sheet thickness $d$, as a model parameter on the product of frequency and conductivity $\omega \sigma$, and on its geometry, so $\Gamma$. They should approximate the exact shielding behaviour, where their accuracy depend on $d, \omega \sigma$ and $\Gamma$ as well.

We will introduce the notion of robustness of impedance transmission conditions which we want to make clear on two examples. An overview of the studied ITCs with respect to their robustness is given in Table 3.1 . 
3.1.1. Examples. As a particular example the PEC boundary conditions (see Sect. 2.2.1), which correspond to absolute shielding, depend on nothing. If we fix the geometry of the sheet mid-line $\Gamma$ and its thickness, the accuracy of the PEC will obviously be the better the larger the model parameter $\omega \sigma$, whereas the smaller $\omega \sigma$ the smaller the accuracy will get, and in the limit $\omega \sigma \rightarrow 0$ where the sheet gets non-conducting the behaviour predicted by the PEC is utterly wrong and even does not get better for smaller thicknesses. This is why we call the PEC a non-robust transmission condition.

Contrary, the impedance transmission conditions ITC-1-0, see (2.12), depend on the conductivity $\sigma$ in a way such they adapt weather the shielding is strong (even for the limit $\omega \sigma \rightarrow \infty$ ) or weak (even for the limit $\omega \sigma \rightarrow 0$ ). To see this, we rewrite $(2.12 \mathrm{~b})$ in terms of the electric field

$$
\left[\operatorname{curl}_{2 D} e_{\mathrm{ITC}, 1,0} \cdot \mathbf{n}^{\perp}\right]=-i d \omega \mu_{0} \sigma e_{\mathrm{ITC}, 1,0} .
$$

At finite energies both sides of the equation are bounded, and hence, for $\omega \sigma \rightarrow \infty$ the electric field $e_{\mathrm{ITC}, 1,0} \rightarrow 0$ on $\Gamma$ corresponding to the PEC boundary condition which is a meaningful approximation to the exact conducting sheet in the limit $\omega \sigma \rightarrow \infty$ (Dirichlet boundary condition is once applied at $\Gamma$ and once at the two sheet surfaces at $s= \pm d / 2$ ). In the limit $\omega \sigma \rightarrow 0$ we have $\left[\operatorname{curl}_{2 D} e_{\mathrm{ITC}, 1,0} \cdot \mathbf{n}^{\perp}\right]=0$ corresponding to the case that there is no conducting sheet at all, and hence, it reproduced the exact solution for the non-conducting sheet. As for any fixed value of $\omega \sigma$ the accuracy of ITC-1-0 depends only on the thickness $d$ and since this is the case also for the limits to $\omega \sigma \rightarrow 0$ and $\omega \sigma \rightarrow \infty$ the ITC-1-0 are robust impedance transmission conditions in the meaning of the following definition.

3.1.2. Definition of robustness and asymptotic consistency. Differently to [34] we will use here a more general definition of the robustness which is independently on any asymptotic framework.

DEFINITION 3.1 (Robustness of an impedance transmission condition). We call an impedance transmission condition ITC robust if for a fixed geometry, its accuracy in any sub-domain $G \subset \Omega_{\text {ext }}^{d}$ is only limited by a constant depending on the sheet thickness $d$,

$$
\left\|e-e_{\mathrm{ITC}}\right\|_{H^{1}(G)} \leq C(\Gamma, d)\|e\|_{H^{1}(G)}
$$

while an upper bound $C(\Gamma, d)<1$ for $d$ small enough can be specified independently of the model parameter $\omega \sigma$.

DEFINITION 3.2 (Robust and asymptotically consistent impedance transmission condition). Robust impedance transmission condition are asymptotically consistent if the upper bound $C(\Gamma, d)$ approaches zero for vanishing sheet thickness $d \rightarrow 0$.

REMARK 3.3. For simulations in time-domain where the field consist of contributions from several frequencies $\omega$ the use of robust impedance transmission conditions will become essential.

As for all ITCs there is a continuous dependency on the model parameter $\omega \sigma$ it is enough to study the accuracy for their limits $\omega \sigma \rightarrow 0$ and $\omega \sigma \rightarrow \infty$, and due to the continuous dependency on $d$ even for the limit $d \rightarrow 0$. So the robustness and asymptotic consistency is equivalent to show

$$
\begin{aligned}
\lim _{d \rightarrow 0} \lim _{\omega \sigma \rightarrow 0}\left\|e-e_{\mathrm{ITC}}\right\|_{H^{1}(G)} & =0, \\
\lim _{d \rightarrow 0} \lim _{\omega \sigma \rightarrow \infty}\left\|e-e_{\mathrm{ITC}}\right\|_{H^{1}(G)} & =0 .
\end{aligned}
$$

With $\omega \sigma \rightarrow 0$ and fixed thickness $d$ the sheet gets non-conducting and for $d \rightarrow 0$ the exact electric field $e$ fulfils the continuity conditions in homogeneous media

$$
[e]=\left[\operatorname{curl}_{2 D} e \cdot \mathbf{n}^{\perp}\right]=0
$$


corresponding to the "no sheet" ITC in Table 2.1. As the PDE in the exterior $\Omega_{\text {ext }}^{0}$ is the same for $e$ and $e_{\text {ITC }}$ it is equivalent to show instead of (3.1a) that the ITCs approach (3.2) for the limit $\omega \sigma \rightarrow 0$ followed by $d \rightarrow 0$, and since $\gamma=\sqrt{-\mathrm{i} \omega \mu_{0} \sigma}$ to show that

$$
\lim _{d \rightarrow 0} \lim _{\gamma \rightarrow 0} T_{12}=0, \quad \lim _{d \rightarrow 0} \lim _{\gamma \rightarrow 0} T_{13}=0, \quad \lim _{d \rightarrow 0} \lim _{\gamma \rightarrow 0} T_{22}=0, \quad \lim _{d \rightarrow 0} \lim _{\gamma \rightarrow 0} T_{23}=0 .
$$

With the same arguments it is enough to equivalent instead (3.1b) to show that the ITCs approach the PEC boundary condition for $\omega \sigma \rightarrow \infty$ followed by $d \rightarrow 0$, and so

$$
\lim _{d \rightarrow 0} \lim _{|\gamma| \rightarrow \infty} T_{12}=0, \quad \lim _{d \rightarrow 0} \lim _{|\gamma| \rightarrow \infty} T_{13}=0, \quad \lim _{d \rightarrow 0} \lim _{|\gamma| \rightarrow \infty}\left|T_{22}\right|=\infty, \quad \lim _{d \rightarrow 0} \lim _{|\gamma| \rightarrow \infty}\left|T_{23}\right|<\infty
$$

\begin{tabular}{|c|c|c|}
\hline ITC & Robust & if no, the reasons for a wrong solution \\
\hline PEC b.c. & no & for $\omega \sigma \rightarrow 0$ \\
Shielding element (NTFS) & yes & \\
Thin layer IBC (MB) & yes & \\
ITC-1-0 & yes & for $\omega \sigma \rightarrow \infty$ \\
ITC-1-1 & yes & \\
ITC-1-2 & no & \\
ITC-2-0 & yes & \\
ITC-2-1 & yes & \\
\hline
\end{tabular}

TABLE 3.1

Almost all the studied impedance transmission conditions are robust, and the here listed robust ITCs are asymptotically consistent as well. That means they provide meaningful results for any frequency $\omega$ and conductivity $\sigma$.

3.1.3. Verification for the introduced transmission conditions. In Table 3.1 the robustness of the transmission conditions is enlisted. It is easily verified that for $\gamma \rightarrow 0$ followed by $d \rightarrow 0$ all the above coefficients approach zero, except for the PEC condition which does not depend neither on $\gamma$ nor on $d$. Hence, the PEC condition is not robust and do not provide meaningful approximations if $\omega \sigma$ is too small. Since $\tanh (x) / x \rightarrow 0$ for $|x| \rightarrow \infty$ the first two conditions in (3.3b) are fulfilled for all ITCs in Table 2.1 except the ITC-1-2 for which both $T_{12}$ and $T_{13}$ approach infinity. Furthermore, we find that $\lim _{|\gamma| \rightarrow \infty} T_{22}=\infty$ and $\lim _{d \rightarrow 0} \lim _{|\gamma| \rightarrow \infty}\left|T_{23}\right|=0$ for all introduced ITCs except ITC-1-2 for which the limit is infinity. So, the ITC-1-2 is the only not robust one among the introduced ITCs which does not provide meaningful approximations if $\omega \sigma$ is too large.

Discussion. Why is ITC-1-2 not robust while the others are ?

The transmission conditions ITC-1-2 are constructed on the basis of an asymptotic expansion in the framework of asymptotically constant shielding where the model parameter $\gamma$ is scaled (renamed as $\gamma_{1}(\varepsilon)$ ) with the sheet thickness (renamed as $\varepsilon$ ). Here, ITC-1-2 is the second order model which shows additional terms to ITC-1-0 and ITC-1-1 to improve the accuracy in the asymptotic framework. The coefficients in these additional terms, however, explode for large values of $\gamma$ if in the same time the thickness is kept. In contrast to the other approaches by asymptotic expansion ITCs of higher order can be derived with the risk to loose robustness from a particular order on.

The higher accuracy of the ITCs derived by asymptotic expansion will be clear in the analysis for various sheet thicknesses and skin depths in Sect. 3.2. 

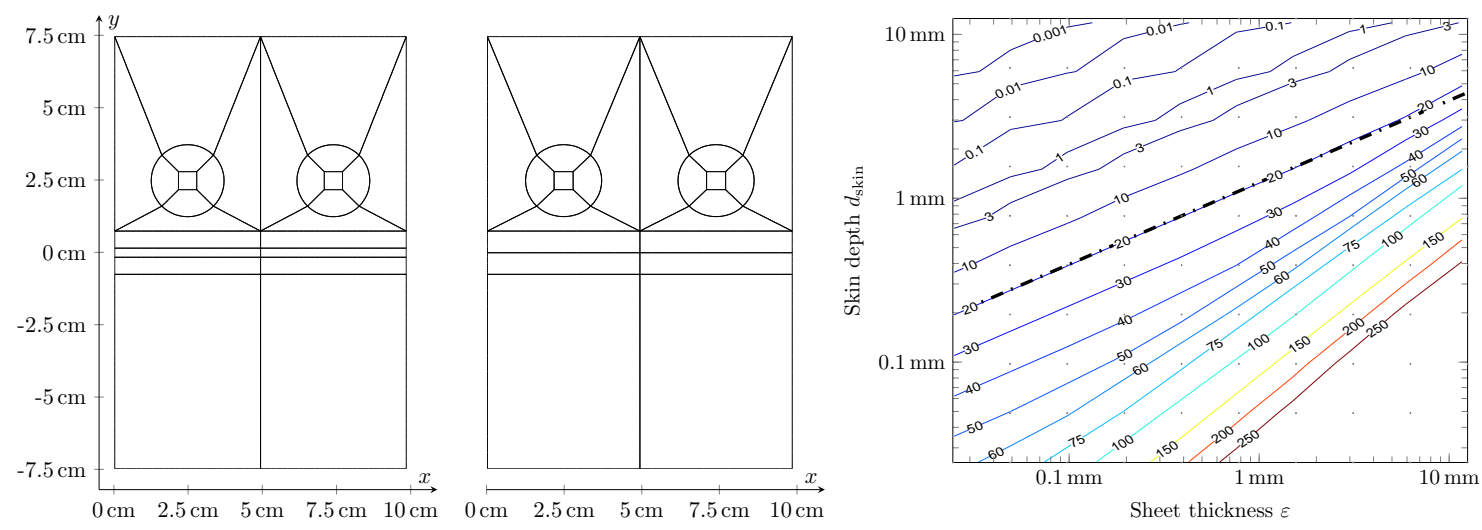

FIG. 3.1. FEM mesh with curved quadrilateral cells for the exact problem with resolved thin sheet of $3.125 \mathrm{~mm}$ thickness (left) and the mesh where the sheet is represented by an interface (middle). We apply periodic boundary conditions on the sides of the rectangular domain and PEC on the top and bottom. To obtain small discretisation errors polynomial degrees of minimally 21 are used. The two elongated cells on both sides of the sheet or interface are not needed in general but ease the comparison of fields computed on the right mesh with fields computed on left mesh. The levels of the shielding efficiencies at position $(2.5 \mathrm{~cm},-2.5 \mathrm{~cm})$ are shown in dependency of sheet thickness and skin depth (right). The shielding is asymptotically constant if the skin depth is proportional to the square root of the sheet thickness, which is indicated by the dash-dotted line $d_{\text {skin }}=\sqrt{\varepsilon \cdot 1.56 \mathrm{~mm}}$ where the shielding efficiency is close to $20.2 \mathrm{~dB}$. The contour lines are interpolated around $10 \times 10$ computed values indicated by tiny points.

3.1.4. Illustration for the example of a straight sheet. For an illustration of the differences in the accuracy of the various transmission conditions we consider a straight sheet of thickness $d=3 \mathrm{~mm}$ in the middle of a rectangular domain of length $10 \mathrm{~cm}$ in $x$ and length $15 \mathrm{~cm}$ (for contour plots) or $20 \mathrm{~cm}$ (for the field plots) in $y$, respectively. We assume periodicity of the domain in direction $x$ and a PEC at the bottom and top boundaries. The periodic boundary conditions on the sides are used to have high regularity and not a singularity in derivatives on some order to be able to investigate the transmission conditions on an example they are constructed for. The sheet is out of copper with conductivity $\sigma=5.91 \cdot 10^{7} \mathrm{~A}(\mathrm{Vm})^{-1}$ where for the frequency of $50 \mathrm{~Hz}(\omega=314 \mathrm{rad} / \mathrm{s})$ the skin depth is $d_{\text {skin }}=9.26 \mathrm{~mm}$, so about 3 times larger than the sheet thickness, and for a frequency of $4 \mathrm{kHz}(\omega=25.1 \mathrm{kHz})$ the skin depth is $d_{\text {skin }}=1.035 \mathrm{~mm}$, about 3 times smaller. We assume a uniform (impressed) current flowing in two circular wires (not self-consistent model by assuming no conductivity in the wire) of radius $1.25 \mathrm{~cm}$ with mid-points at $x=2.5 \mathrm{~cm}$ and $x=7.5 \mathrm{~cm}$ and both at $y=2.5 \mathrm{~cm}$. The current density $j_{0}$ is regulated to have a constant value $i \omega \mu_{0} j_{0}=1 \mathrm{Vm}^{-3}$ in the left wire and $\mathrm{i} \omega \mu_{0} j_{0}=-1 \mathrm{Vm}^{-3}$ in the right one, when changing the frequency. This enables us to compare absolute field values. This is, however, not important for the error analysis as we studied relative errors.

For the above mentioned frequencies we computed the exact fields by resolving the thin sheet and the surrounding by finite elements of high order using the numerical $\mathrm{C}++$ library Concepts [16, 12] where in and around the circular wires coarse curved cells have been used (see Fig. 3.1). The fields for the transmission conditions are computed on a similar mesh in which the sheet is only represented by an interface. In all the computations a polynomial degree minimally of 21 has been chosen such that the discretisation error is negligible over the modelling error introduced by the transmission conditions.

In Table 3.2 the shielding efficiencies at position $(x, y)=(2.5 \mathrm{~cm},-2.5 \mathrm{~cm})$ are evaluated. The low frequency, low shielding case is best approximated by the five transmission conditions derived by asymptotic expansions, where increasing the order increases the accuracy remarkably. For both the shielding element (NTFS) and the thin layer impedance boundary conditions (MB) 


\begin{tabular}{lcc} 
& $f=50 \mathrm{~Hz}$ & $f=4 \mathrm{kHz}$ \\
\hline Exact & $1.053 \mathrm{~dB}$ & $38.77 \mathrm{~dB}$ \\
\hline PEC & $\infty$ & $\infty$ \\
NTFS & $1.784 \mathrm{~dB}$ & $32.95 \mathrm{~dB}$ \\
MB & $2.024 \mathrm{~dB}$ & $40.40 \mathrm{~dB}$ \\
ITC-1-0 & $1.172 \mathrm{~dB}$ & $32.95 \mathrm{~dB}$ \\
ITC-1-1 & $1.042 \mathrm{~dB}$ & $42.35 \mathrm{~dB}$ \\
ITC-1-2 & $1.054 \mathrm{~dB}$ & $17.45 \mathrm{~dB}$ \\
ITC-2-0 & $1.040 \mathrm{~dB}$ & $22.19 \mathrm{~dB}$ \\
ITC-2-1 & $1.048 \mathrm{~dB}$ & $38.72 \mathrm{~dB}$ \\
\hline
\end{tabular}

TABLE 3.2

The shielding efficiencies are approximated to different accuracies by the introduced transmission conditions and for relatively large (first row) and relatively small (second row) skin depths. The numbers are for the problem introduced in Sect. 3.1.4 and expected to be accurate in all given digits.

the shielding efficiency for this case is over-estimated by almost $1 \mathrm{~dB}$. For the higher frequency, high shielding case the shielding efficiency is approximated well by the MB, by the ITC-1-1 and to a surprising accuracy by the ITC-2-1. Both the ITC-1-0 and the shielding element (NTFS) underestimates the shielding efficiency by about $6 \mathrm{~dB}$. By even $16 \mathrm{~dB}$ and $21 \mathrm{~dB}$ the shielding efficiency is underestimated by the ITC-2-0 and ITC-1-2. The poor accuracy for ITC-1-2 for $4 \mathrm{kHz}$ in spite of the very high accuracy for $50 \mathrm{~Hz}$ is another sign of the lack of robustness. Despite its robustness the ITC-2-0 obtains rather low accuracy for $4 \mathrm{kHz}$.

In Fig. 3.2 the exact magnetic fields for the two frequencies and those approximated by the eight introduced ITCs are shown. For the comparison the exterior field originally defined up to the interface is taken only for $|y|>\frac{d}{2}$, and for $|y|<\frac{d}{2}$ the a-posteriori computed interior fields are used. Note, that the approximate fields are discontinuous over $|y|=\frac{d}{2}$, where the jumps are small for small sheet thicknesses.

3.2. Accuracy in dependence of sheet thickness and skin depth. Whether a ITC is robust or not is only a rough decision guidance. In this section we are going to study the accuracy more precisely by repeating the above experiment, but varying the sheet thickness as well as the frequency, and with the latter the skin depth.

We chose ten sheet thicknesses starting from $12.5 \mathrm{~mm}$ and taking always the respective half till $0.0244 \mathrm{~mm}$. These thicknesses we combined with the same ten values for the skin depths such that we have of skin depth to thickness ratios of $1 / 1000$ to 1000 to cover the relevant cases and come even so close to limit behaviours. We used the mesh in Fig. 3.1 (left) while varying the thickness of the cells in and around the sheet for the computation of reference solutions, i. e., very accurate approximations to the exact electric and magnetic fields. To obtain those we used minimally polynomial degrees of 42 in all cells which exactly resolve the thin sheet and the circular wires. Inside the sheet we increased the polynomial degree anisotropically in thickness direction the more the smaller skin depth to thickness ratios, this is up to 198. On the mesh in Fig. 3.1 (middle) where the thin sheet is represented by an interface only we have computed the electric and magnetic fields with the introduced ITCs. We have chosen also quite high polynomial degree of 26 such that the discretisation error is mostly negligible over the modelling error. For example the relative discretisation error of the magnetic field measured in the $L^{2}$-norm in the exterior of the sheet is about $3 \cdot 10^{-7}$. In the numerical experiments we measure the discretisation and modelling error together and have chosen by purpose the polynomial degree smaller than those for the reference solution to identify easily the parameter range where the plateau of a dominating discretisation 


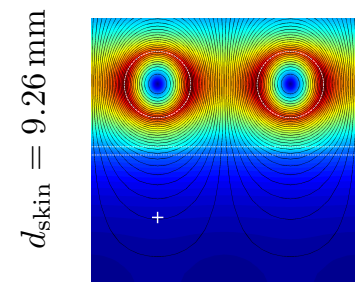

Exact

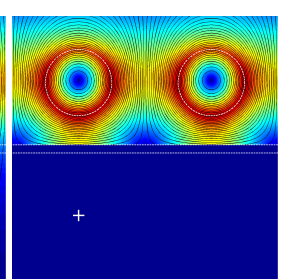

PEC

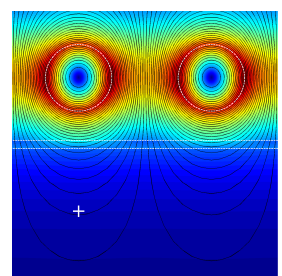

ITC-1-1

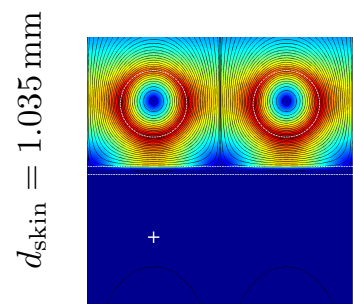

Exact

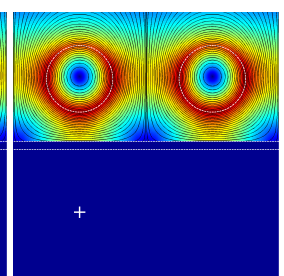

PEC

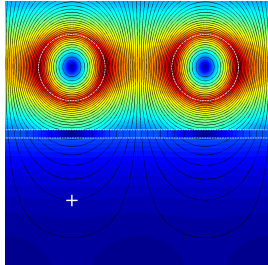

NTFS

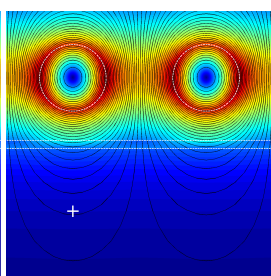

ITC-1-2

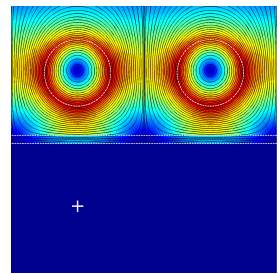

ITC-1-1

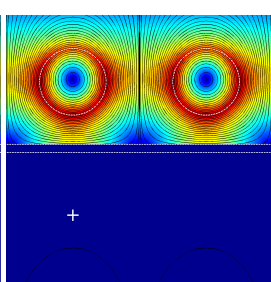

NTFS

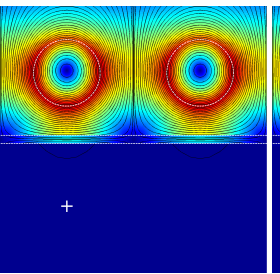

ITC-1-2

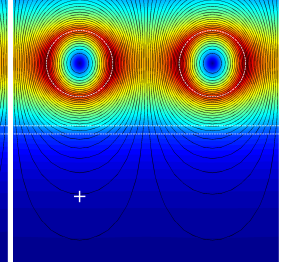

MB

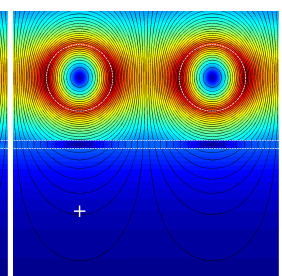

ITC-2-0

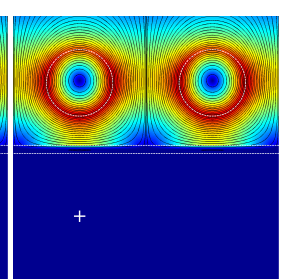

MB

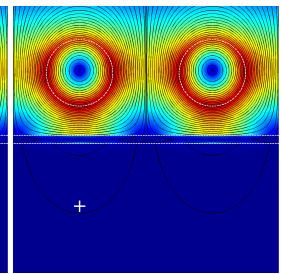

ITC-2-0

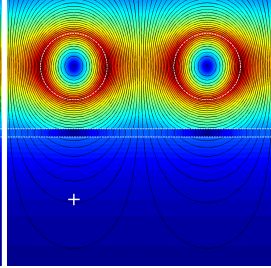

ITC-1-0

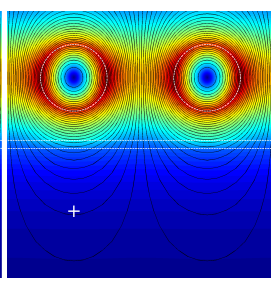

ITC-2-1

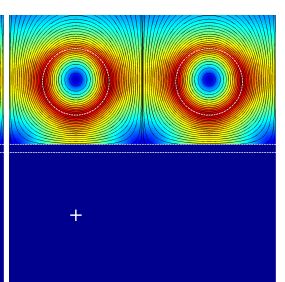

ITC-1-0

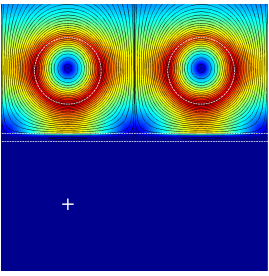

ITC-2-1

FIG. 3.2. The magnitude and the flux lines of the in-plane magnetic field for the shielding of two live wires by a copper sheet of thickness $d=3 \mathrm{~mm}$ at $50 \mathrm{~Hz}$ and $4 \mathrm{kHz}$ for which the skin depth is $9.26 \mathrm{~mm}$ and $1.035 \mathrm{~mm}$ (fields are shown only up to $|y|=5 \mathrm{~cm})$. In the upper left corner the results for the exact model is shown, where those of the eight introduced transmission conditions follow. The point for evaluation of the shielding efficiency is indicated by white crosses.

error is reached.

In Fig. 3.3 the relative modelling error of the magnetic field in the exterior of the sheet for the introduced transmission conditions is illustrated in contour line plots. Note that the relative discretisation error is more than $30 \%$ below the lowest level contour of $5 \cdot 10^{-7}$. In the following we will discuss the results for the different transmission conditions individually.

PEC. The accuracy of the perfect electric conductor boundary condition depends as expected from the skin depth to thickness ratio. However, surprisingly it is the best for a ratio of about $1 / 2$ and decays for both, lower and higher values. For skin depth to thickness ratio lower than $1 / 10$ the accuracy is very close to those of the shielding element (NTFS), the Mayergoyz-Bedrosian conditions and as well as ITC-1-0, ITC-1-1 and ITC-2-0, and depends on the thickness only. In this (parameter) area the exact fields inside and behind the sheet are almost zero and the error 


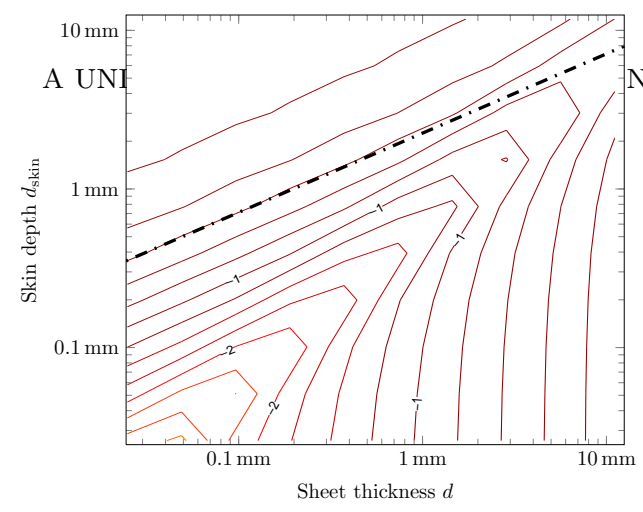

(a) PEC.

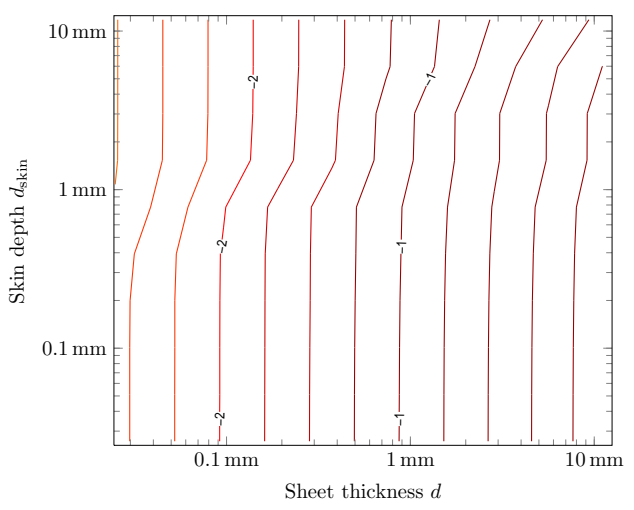

(c) Mayergoyz-Bedrosian conditions.

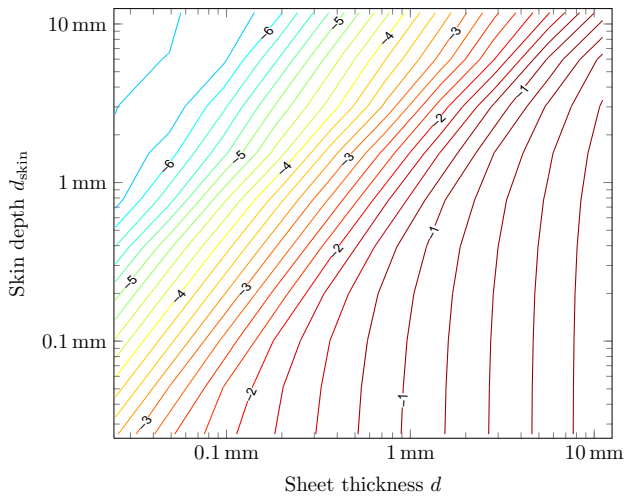

(e) ITC-1-1.

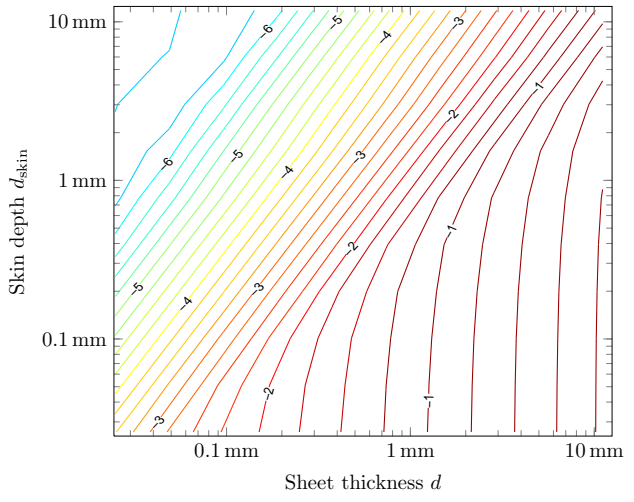

(g) ITC-2-0.

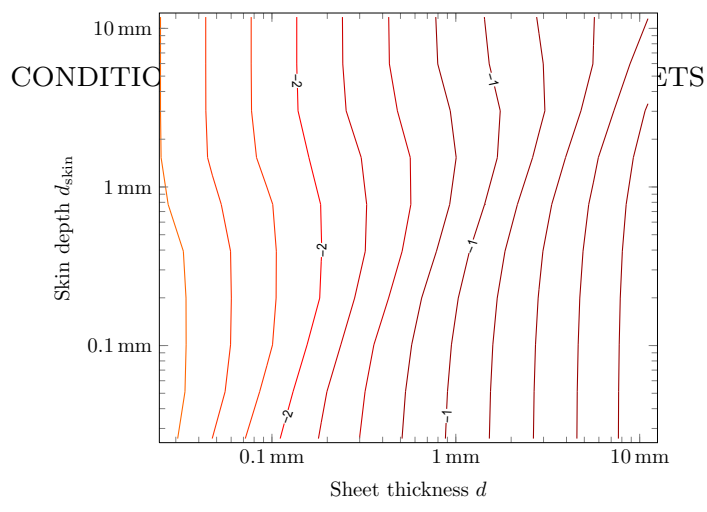

(b) NTFS.

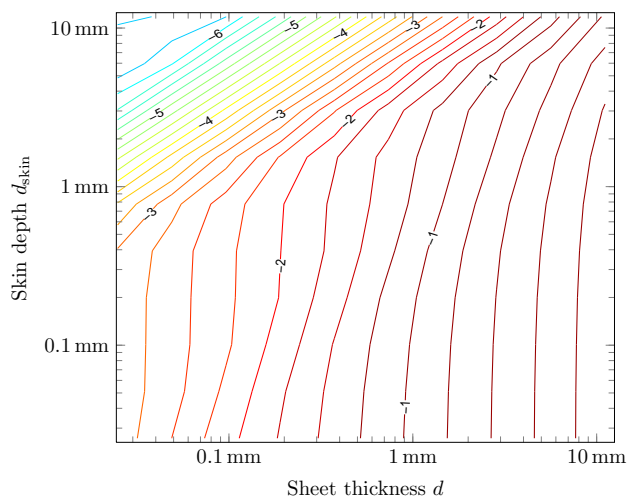

(d) ITC-1-0

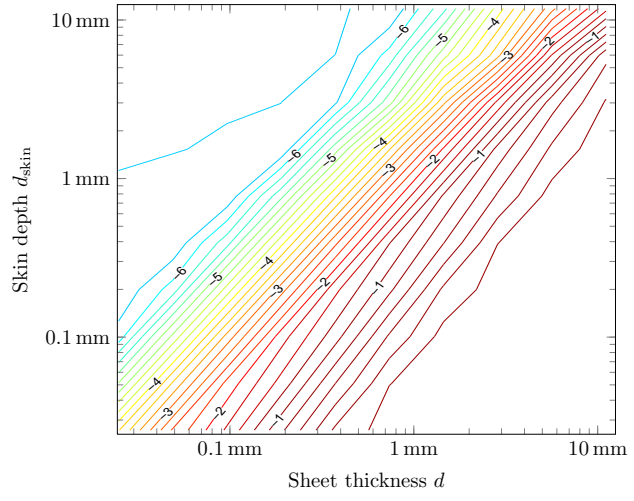

(f) ITC-1-2.

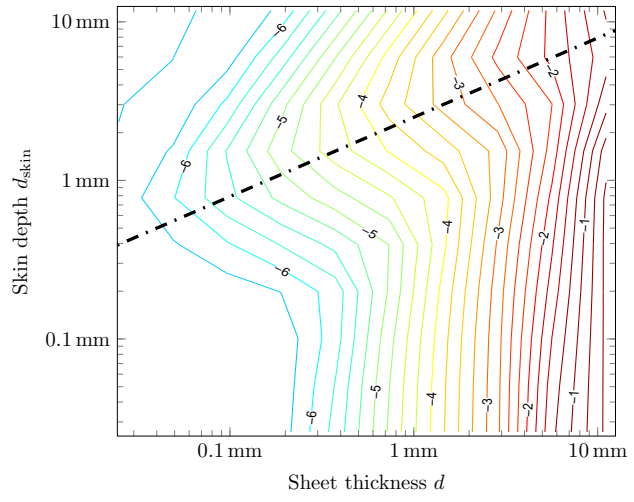

(h) ITC-2-1.

FIG. 3.3. Contour plots of the relative magnetic field modelling error $\left.\left\|\mathbf{h}-\mathbf{h}_{\mathrm{ITC}}\right\|_{L^{2}\left(\Omega_{\text {ext }}^{d}\right)}\right)\|\mathbf{h}\|_{L^{2}\left(\Omega_{\text {ext }}^{d}\right)}$ in the exterior of straight copper sheets (see Sect.3.1.4) for the introduced transmission conditions where both the sheet thickness and skin depth are computed for $12.5 \mathrm{~mm}, 6.25 \mathrm{~mm}, 3.125 \mathrm{~mm}, \ldots, 0.0244 \mathrm{~mm}$. 
comes from the fact that the PEC is placed at the mid-line rather than at $\pm \frac{d}{2}$. If the skin depth to thickness ratio is above 2 wrong predicted fields behind the sheet dominate the error which depends on the ratio $d_{\text {skin }} / \sqrt{d}$.

NTFS and MB. The shielding element (NTFS) [29] and the thin layer impedance boundary conditions by Mayergoyz and Bedrosian [27] have a different appearance and are derived by alternative assumptions. However their accuracy is pretty the same in the whole parameter area (up to some small factors). Interestingly, the accuracy depends on the sheet thickness only and does not provide better results for either large or small skin depth to thickness ratios.

ITC-1-0, ITC-1-1 and ITC-2-0. The impedance transmission conditions derived by asymptotic expansion of order 0 and 1 have at least the same accuracy than NTFS and MB and provide higher accuracy for large skin depth to thickness ratios. This extra accuracy is observed for ITC1-1 in a much larger parameter area than for ITC-1-0. Despite its different derivation the ITC-2-0 show almost the same accuracy levels than ITC-1-1 in the whole parameter area.

ITC-1-2. We have seen in Sect. 3.1 that differently to ITC-1-0 and ITC-1-1 the second order impedance transmission condition ITC-1-2 is not robust for large frequencies which is visible in an error increase for low skin depth to thickness ratios. On the other hand, if the skin depth is at the order of the thickness or larger the accuracy is much improved over the above mentioned ITCs.

ITC-2-1. The ITC-2-1 are the only transmission conditions that omit a remarkable higher accuracy than PEC for smaller skin depths than thicknesses. For very low ratios the accuracy is comparable to that of ITC-1-1 (or ITC-2-0) which is constructed for this area. The ITC-2-1 provide accurate results for both, large and small, frequencies and are therefore robust. The lowest accuracy has been observed around $d_{\text {skin }}=\sqrt{d \cdot 6.25 \mathrm{~mm}}$ (see dash-dotted line in Fig 3.3(h)).

\begin{tabular}{|l|ccc|}
\cline { 2 - 4 } \multicolumn{1}{c|}{} & \multicolumn{3}{c|}{ Asymptotic regime } \\
\multicolumn{1}{c|}{} & $-0-$ & $-1-$ & $-2-$ \\
\hline PEC & - & - & 0 \\
NTFS & 0 & 0 & 0 \\
MB & 0 & 0 & 0 \\
ITC-1-0 & 2 & 0 & 0 \\
ITC-1-1 & 2 & 1 & 0 \\
ITC-1-2 & 3 & 2 & 0 \\
ITC-2-0 & 2 & 1 & 0 \\
ITC-2-1 & 2 & 1 & $1(2)$ \\
\hline
\end{tabular}

TABLE 3.3

The asymptotic order of the introduced ITCs in different asymptotic regimes. Note, that a model order $N$ converge like $O\left(d^{N+1}\right)$ to the exact model. No convergence is indicated by "-". The number in brackets is the order which we observed numerically for a straight sheet.

3.3. Asymptotic expansion of the transmission conditions. All the transmission conditions are derived to obtain better results with decreasing sheet thickness. Looking for asymptotically small sheet thicknesses those transmission conditions derived by asymptotic expansions are optimal in their own asymptotic regime. The most relevant asymptotic regimes are

-0 - to fix the frequency and so the skin depth,

- 1 - the asymptotically constant shielding with $d_{\text {skin }} \sim 1 /|\gamma| \sim \sqrt{d}$ (see Sect. 2.2 .4 and Fig. 3.1 (right)), and

- 2 - the asymptotically constant relative skin depth with $d_{\text {skin }} \sim d$ (see Sect. 2.2.5). 
To illustrate of the asymptotic regime 1 we have computed the shielding efficiencies at position $(2.5 \mathrm{~cm},-0.25 \mathrm{~cm})$ for the example of the straight sheet introduced in Sect. 3.1.4. We have computed on the exact model with resolved copper sheet with polynomial degrees of 43 and increased polynomial degrees in thickness direction depending of the ratio of skin depth and thickness. The shielding efficiencies are shown in a contour plot in Fig. 3.1 (right) in dependence of the sampled thicknesses and frequencies like in Sect. 3.2.

The transmission conditions ITC-1-N are by construction optimal in the regime 1 , i. e., they are of order $N$ (the modelling error converges like $O\left(d^{N+1}\right)$. For fixed frequency (regime 0 ) we observe that they are even of order 2 for $N=0,1$ (error $O\left(d^{3}\right)$ ) and that they are of order 3 for $N=2$ (error $O\left(d^{4}\right)$ ) by comparing the terms in $T_{i j}$ with those in the respective next order model (see [37] for a definition of ITC-1-3). In asymptotic regimes 0 and 1 the PEC does not converge to the right limit at all. The shielding element has a wrong term $d \partial_{\Gamma}^{2}$ in comparison to the asymptotically optimal ITC-1-0 and ITC-1-1 which retains it at order 0 in both regimes. The asymptotic expansions of the (non-constant) terms of the Mayergoyz-Bedrosian conditions for constant frequency (regime 0) are

$$
\begin{aligned}
& T_{13}=-\frac{2}{\gamma} \tanh \left(\gamma \frac{d}{2}\right)=-d+O\left(d^{3}\right)=\frac{\gamma^{2} d^{3}}{12}+O(d), \\
& T_{22}=-2 \gamma \tanh \left(\gamma \frac{d}{2}\right)=-\gamma^{2} d+O\left(d^{3}\right),
\end{aligned}
$$

where we used the Taylor expansion of tanh. The term $T_{13}$ differs from the optimal condition ITC-1-0 by $O(d)$ and gets only linear convergence in the regime 0 . This is also the case in regime 1 as the wrong term $-d$ does not depend on $\gamma$. In difference we have for ITC-2-0

$$
T_{22}=-\frac{2 \gamma \sinh \left(\gamma \frac{d}{2}\right)}{\cosh \left(\gamma \frac{d}{2}\right)-\gamma \frac{d}{2} \sinh \left(\gamma \frac{d}{2}\right)}=-\gamma^{2} d\left(1+\frac{\gamma^{2} d^{2}}{6}+\frac{7 \gamma^{4} d^{4}}{240}\right)=-\gamma^{2} d\left(1+\frac{\gamma^{2} d^{2}}{6}\right)+\gamma^{6} O\left(d^{5}\right),
$$

and the order is the same as for ITC-1-1. For ITC-2-1 this term is the same and the other terms of the first order transmission condition ITC-2-1 are equal to those of ITC-1-1 up to $O\left(\gamma^{4} d^{5}\right)$. The transmission conditions ITC-2-N are by construction optimal in the regime 2 . Nevertheless, in the other regimes their convergence is like the order 1 model ITC-1-1.

The regime 2 is special as the optimal conditions have terms with $\sinh \left(\gamma \frac{d}{2}\right)$ and $\cosh \left(\gamma \frac{d}{2}\right)$ which cannot be recovered by any finite order polynomial in $\gamma$ and $d$. From the introduced transmission conditions only ITC-2-1 obtains in this regime a quadratic convergence. The real limit condition in this regime is the PEC and so it make sense to study asymptotically the quotients $T_{21} / T_{22}$ and $T_{23} / T_{22}$ which are zero for PEC. For ITC-2-0 we have $T_{23} / T_{22}=0$ as well and with the constant $c=\gamma \frac{d}{2}$ we have

$$
\frac{T_{21}}{T_{22}}=-\frac{\cosh (c)-c \sinh (c)}{4 c \sinh (c)} d=O(d),
$$

and so ITC-2-0 convergences as PEC linearly in the thickness as well. The same holds for the other introduced transmission conditions, where we will not give the proof here. We have collected all the orders of convergence in Tab. 3.3.

To illustrate the different order of the ITCs in the three asymptotic regimes we have drawn the error in the magnetic field in the exterior of the sheets along one line in each asymptotic regime, see Fig. 3.4. The example is again the straight copper sheet described in Sect. 3.1.4, and the results are taken from Sect. 3.2. We observe the same convergence orders for electric field outside the sheet and for both the electric and magnetic field they are shifted by $1 / 2$ due to the shrinking area of the sheet for decreasing thickness, which we, however, do not show here (see e.g. [37] for ITC-1-N). 
PEC. The error does not converge to zero for constant skin depth $d_{\text {skin }}$ and $d_{\text {skin }} \sim \sqrt{d}$ which manifests the lack of robustness. For constant skin depth there is a linear convergence in the sheet thickness.

NTFS and MB. In all three asymptotic regimes the transmission conditions of Nakata et.al. and those of Mayergoyz and Bedrosian show linear convergence in $d$.

ITC-1-0. Linear convergence obtains ITC-1-0, the simplest impedance transmission condition obtained by asymptotic expansion, in all asymptotic regimes except for constant skin depth where we justify numerically the predicted cubic convergence in $d$.

ITC-1-1 and ITC-2-0. Derived by asymptotic expansions for asymptotically constant shielding, $i$. $e ., d_{\text {skin }} \sim \sqrt{d}$, the ITC-1-1 leads to quadratic convergence in this regime. The convergence order in the other two regimes is the same as for ITC-1-0, only the constant improves. We see also here, that the ITC-2-0 does not differ from ITC-1-1 in the order, but only by the constant.

ITC-1-2. The highest convergence order in the first two asymptotic regimes is obtained by the ITC-1-2 where for constant relative skin depth the order remains 1 with an even improved constant over ITC-1-1. This shows that ITC-1-2 is preferable for applications where the frequency is so low that the skin depth is comparable or larger than the sheet thickness.

ITC-2-1. This transmission condition is the only one with higher convergence order for constant relative skin depth. We observe for the straight sheet cubic convergence (order 2) where an order of 1 is predicted. For other asymptotic regimes the order of convergence is the same as ITC-1-1 the other conditions derived by asymptotic expansions at order 1 .

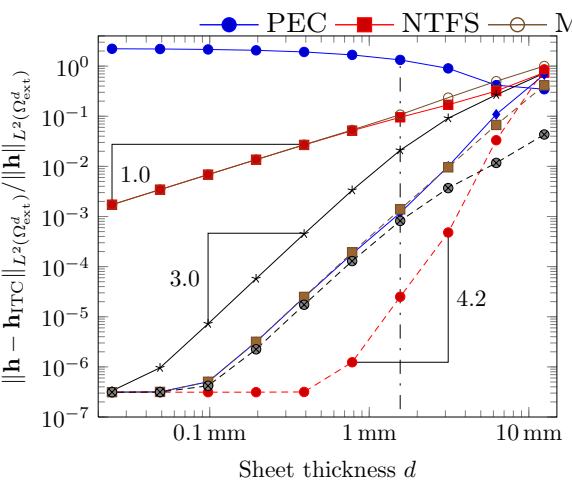

(a) Convergence for $d_{\text {skin }}=1.56 \mathrm{~mm}$.

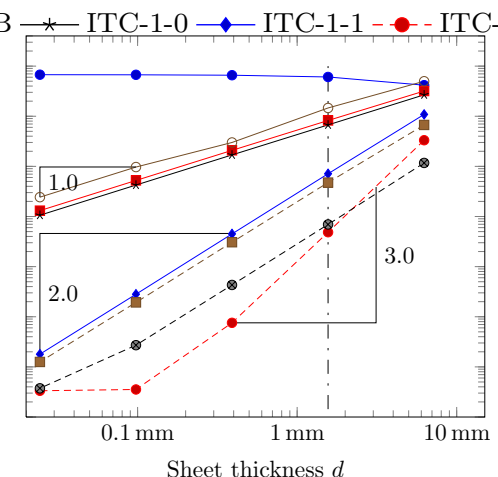

(b) Convergence for $d_{\text {skin }}=\sqrt{d \cdot 1.56 \mathrm{~mm}}$

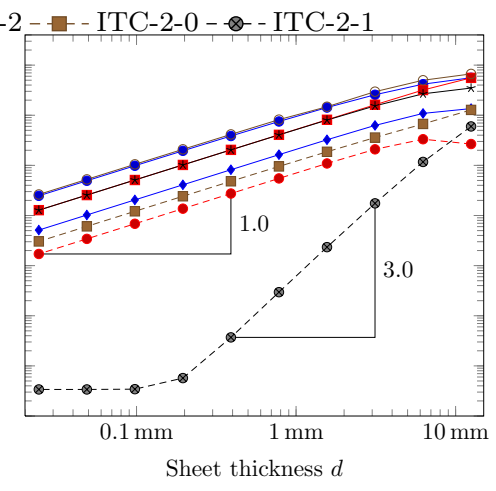

(c) Convergence for $d_{\text {skin }}=d$.

FIG. 3.4. The relative magnetic field modelling error in the exterior of the straight copper sheets (see Sect. 3.1.4) for the introduced transmission conditions, which is shown in three asymptotic frameworks. In (a) the frequency and so the skin depth is fixed while the sheet thickness is varied. In (b) the skin depth is varied like the square root of the thickness. For the asymptotics (a) and (b) the sheet thickness for which the skin depth is equal is shown by a dash-dotted line. In (c) the skin depths is varied together with the sheet thickness. The order of the modelling errors in the sheet thickness depends on the asymptotic regime.

4. Discussion. We have shown that the thin layer impedance boundary conditions of Mayergoyz et.al. applied on the sheet mid-line and the shielding element of Nakata et.al. are robust with respect to the frequency and valuable improvements over the simple perfect electric conductor boundary condition. They may be, however, easily replaced by the ITC-1-0, the simplest impedance transmission condition derived by asymptotic expansions without any loss of accuracy and even accuracy improvement for very low shielding. This replacement recommended by the achieved accuracy is also supported by the very simple structure of ITC-1-0 with continuity of 
the electric field like for the shielding element, i.e., no node doubling in a finite element or finite difference code, and with even one term less than the shielding element. The same simple structure is shown by the ITC-1-1 and the ITC-2-0 which improve in the case of not too small skin depth to thickness ratio the accuracy of ITC-1-0 remarkably. The ITC-1-2 are specially suited for low frequency applications where the skin depths does not exceed the sheet thickness and where they show much higher accuracy than the other ITCs. However, the ITC-1-2 appear to possess the most complex structure, first, the electric field is not continuous over the interface, second, the condition for the electric field jump makes a mixed formulation necessary, and third, a second tangential derivative like for the shielding element and terms with the curvature are involved. For a descent accuracy under all conditions the use of ITC-1-1 and ITC-2-0 is recommended. For high accuracy requirements under all conditions the ITC-2-1 are the only ones of the introduced which can be applied. An implementation of a thin layer impedance boundary condition can be changed to ITC-2-1 for straight sheets by simply exchanging the coefficients. For curved sheets there are a few additional terms involving the curvature, but no tangential derivatives.

The comparison in this article is for transmission conditions on the sheet mid-line $\Gamma$. Motivated by the results in [33, Part II] we believe that the conditions by Mayergoyz et.al. obtain higher accuracies when the jumps and mean values are applied on both sides of the sheet, which have to be resolved in the mesh.

Conclusion. In this article eight impedance transmission conditions for thin conducting sheets in the eddy current model are systematically analysed and compared with respect to their robustness, their accuracy in dependence of the important parameters thickness and skin depth, and their asymptotic expansion in the three relevant asymptotic regimes. Two of this transmission conditions, called ITC-2-0 and ITC-2-1, have been proposed first time in this article which are products of asymptotic expansions for asymptotically constant skin-depth to thickness ratio [34]. All the transmission conditions are well integrated into variational formulations for an approximative solution in the exterior of the mid-line of the thin sheet, which turn out to be well-posed, and are therefore well-suited to use within the finite element method. For all transmission conditions we specified formulas to evaluate the field in the sheet in a post-processing step. Six of the transmission conditions are robust with respect to the frequency, meaning that they can be used for all frequencies, where the accuracy gets the better the smaller the sheet thickness. Only the PEC boundary condition leads to utterly wrong results for high skin-depth to thickness ratios and the ITC-1-2 does not predict the fields accurately if this ratio is high. Analysing the accuracy in dependence of sheet thickness and skin depth as well as their asymptotic expansion we found that the shielding element and the thin layer impedance boundary condition have very similar results, even so their appearance is different, as well as ITC-1-1 and ITC-2-0. If very accurate results under all conditions are demanded the ITC-2-1 shall be used where exclusively for high skin-depth to thickness ratio the ITC-1-2 behave best. For lower demands on accuracy the ITC-1-1 or ITC-2-0 are preferable to use due to their simpler structure.

\section{REFERENCES}

[1] Antoine, X., BarucQ, H., And Vernhet, L. High-frequency asymptotic analysis of a dissipative transmission problem resulting in generalized impedance boundary conditions. Asymptot. Anal. 26, 3-4 (2001), 257-283.

[2] Bartoli, N., And Bendali, A. Robust and high-order effective boundary conditions for perfectly conducting scatterers coated by a thin dielectric layer. IMA J. Appl. Math. 67 (2002), 479-508.

[3] Bateman, H. The Mathematical Analysis of Electrical and Optical Wave-Motion on the basis of Maxwell's Equations. Cambridge University Press, 1915.

[4] Bendali, A., and Lemrabet, K. The effect of a thin coating on the scattering of a time-harmonic wave for the Helmholtz equation. SIAM J. Appl. Math. 6 (1996), 1664-1693.

[5] Bendali, A., And Lemrabet, K. Asymptotic analysis of the scattering of a time-harmonic electromagnetic wave by a perfectly conducting metal coated with a thin dielectric shell. Asymptot. Anal. 57, 3 (2008), 
$199-227$.

[6] Biro, O., Preis, K., Richter, K., Heller, R., Komarek, P., and Maurer, W. Fem calculation of eddy current losses and forces in thin conducting sheets of test facilities for fusion reactor components. IEEE Trans. Magn. 28, 2 (1992), 1509-1512.

[7] Bottauscio, O., Chiampi, M., and Manzin, A. Transient analysis of thin layers for the magnetic field shielding. IEEE Trans. Magn. 42, 4 (April 2006), 871-874.

[8] Caloz, G., Costabel, M., Dauge, M., and Vial, G. Asymptotic expansion of the solution of an interface problem in a polygonal domain with thin layer. Asymptot. Anal. 50, 1 (2006), 121-173.

[9] Carpenter, C., And Duurovi'c, M. Three-dimensional numerical solution of eddy currents in thin plates. Proc. IEE 122, 6 (1975), 681-688.

[10] Celozzi, S., Lovat, G., And Araneo, R. Wiley Encyclopedia of Electrical and Electronics Engineering. John Wiley \& Sons, Inc., 2001, ch. Electromagnetic Shielding.

[11] Ciuperca, I., Perrussel, R., and Poignard, C. Two-scale analysis for very rough thin layers. an explicit characterization of the polarization tensor. J. Math. Pures Appl. 95, 3 (2011), 277-295.

[12] Concepts Development Team. Webpage of Numerical C++ Library Concepts 2. http://www.concepts.math.ethz.ch, 2012.

[13] Davies, E. Conduction and induction heating. IEE Power Engineering Series II. Peter Peregrinus Ltd., London, UK, 1990

[14] Duruflé, M., Haddar, H., and Joly, P. Higher order generalized impedance boundary conditions in electromagnetic scattering problems. C. R. Physique 7, 5 (2006), 533-542.

[15] Engquist, B., And NÉDÉLEC, J.-C. Effective boundary conditions for acoustic and electromagnetic scattering in thin layers. Tech. rep., Ecole Polytechnique Paris, 1993. Rapport interne du C.M.A.P.

[16] Frauenfelder, P., and Lage, C. Concepts - An Object-Oriented Software Package for Partial Differential Equations. Math. Model. Numer. Anal. 36, 5 (2002), 937-951.

[17] Guérin, C., Tanneau, G., Meunier, G., Labie, P., Ngnegueu, T., and Sacotte, M. A shell element for computing 3D eddy currents - Application to transformers. IEEE Trans. Magn. 31, 3 (1995), 1360-1363.

[18] Gyselinck, J., Sabariego, R., Dular, P., and Geuzaine, C. Time-domain finite-element modeling of thin electromagnetic shells. IEEE Trans. Magn. 44, 6 (2008), $742-745$

[19] Haddar, H., ANd Joly, P. Stability of thin layer approximation of electromagnetic waves scattering by linear and nonlinear coatings. J. Comput. Appl. Math. 143, 2 (2002), 201-236.

[20] Haddar, H., Joly, P., And NGuyen, H. Generalized impedance boundary conditions for scattering by strongly absorbing obstacles: the scalar case. Math. Models Methods Appl. Sci 15, 8 (2005), 1273-1300.

[21] Igarashi, H., Kost, A., And Honma, T. Impedance boundary condition for vector potentials on thin layers and its application to integral equations. Eur. Phys. J. AP 1 (1998), 103-109.

[22] Jin, J.-M., Volakis, J. L., YU, C., AND Woo, A. Modeling of resistive sheets in finite element solutions [em scattering]. IEEE Trans. Antennas and Propagation 40, 6 (1992), 727-731.

[23] Knott, E., And Senior, T. Non-specular radar cross section study. Tech. Rep. 011764-1-T, Univ. Michigan. Radiation Lab., Ann Arbor, MI, USA, Jan 1974.

[24] KRÄhenbühl, L., AND MulleR, D. Thin layers in electrial engineering. Example of shell models in analysing eddy-currents by boundary and finite element methods. IEEE Trans. Magn. 29 (1993), 1450-1455.

[25] Leontovich, M. A. On approximate boundary conditions for electromagnetic fields on the surface of highly conducting bodies (in russian). Research in the propagation of radio waves (1948), 5-12. Moscow, Academy of Sciences.

[26] Levi-Civita, T. La teoria elettrodinamica di Hertz di fronte ai fenomeni di induzione. Rend. Lincei (5) 11, 2 (1902), 75-81. (in Italian).

[27] Mayergoyz, I., and Bedrosian, G. On calculation of 3-D eddy currents in conducting and magnetic shells. IEEE Trans. Magn. 31, 3 (1995), 1319-1324.

[28] MCWhirter, J. Computation of three-dimensional eddy currents in thin conductors. Magnetics, IEEE Transactions on 18, 2 (1982), 456-460.

[29] Nakata, T., Takahashi, N., Fujiwara, K., and Shiraki, Y. 3D magnetic field analysis using special elements. IEEE Trans. Magn. 26, 5 (1990), 2379-2381.

[30] Poignard, C. Approximate transmission conditions through a weakly oscillating thin layer. Math. Meth. Appl. Sci. 32, 4 (2009).

[31] Poltz, J., And Romanowski, K. Solution of quasi-stationary field problems by means of magnetic scalar potential. IEEE Trans. Magn. 19, 6 (1983), 2425-2428.

[32] Rodger, D., And Atkinson, N. Finite element method for 3D eddy current flow in thin conducting sheets. IEE Proceedings A 135, 6 (1988), 369-374.

[33] Schmidt, K. High-order numerical modeling of highly conductive thin sheets. PhD thesis, ETH Zurich, July 2008.

[34] Schmidt, K., and Chernov, A. Robust families of transmission conditions of high order for thin conducting sheets. Tech. Rep. 1102, Institut for Numerical Simulation, University of Bonn, Feb 2011.

[35] Schmidt, K., And Hiptmair, R. Asymptotic boundary element methods for thin conducting sheets in two 
dimensions. Submitted to IEEE Trans. Magn.

[36] Schmidt, K., And Tordeux, S. Asymptotic modelling of conductive thin sheets. Z. Angew. Math. Phys. 61, 4 (2010), 603-626.

[37] Schmidt, K., And Tordeux, S. High order transmission conditions for thin conductive sheets in magnetoquasistatics. ESAIM: M2AN 45, 6 (2011), 1115-1140.

[38] Senior, T. Approximate boundary conditions. IEEE Trans. Antennas and Propagation 29, 5 (1981), 826-829.

[39] Senior, T., and Volakis, J. Approximate Boundary Conditions in Electromagnetics. Institution of Electrical Engineers, 1995.

[40] Shchukin, A. N. Propagation of Radio Waves (in russian). Svyazizdat, Moscow, 1940.

[41] Shewchuk, J. What Is a Good Linear Finite Element? Interpolation, Conditioning, Anisotropy, and Quality Measures. Preprint, University of California at Berkeley, 2002.

[42] Tozoni, O., and Mayergoyz, I. Calculation of three-dimensional electromagnetic fields. Technika, Kiev, 1974. in russian.

[43] Trommler, J., Koch, S., and Weiland, T. A finite-element approach in order to avoid ill-conditioning in thin-sheet problems in frequency domain - application to magneto-quasistatics. J. Comput. Appl. Math. 236, 18 (2012), $4671-4680$. 